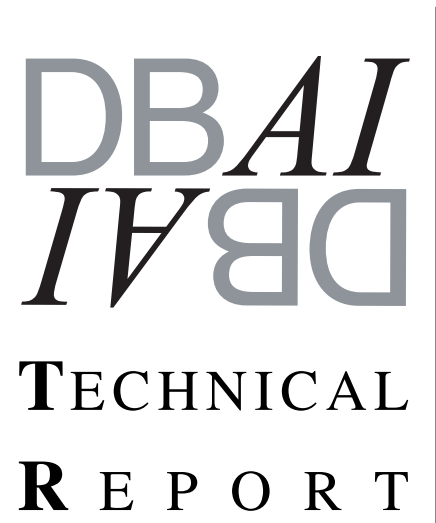

Institut für Informationssysteme

Abteilung Datenbanken und

Artificial Intelligence

Technische Universität Wien

Favoritenstr. 9

A-1040 Vienna, Austria

Tel: $\quad+43-1-58801-18403$

Fax: $\quad+43-1-58801-18492$

sekret@dbai.tuwien.ac.at

www.dbai.tuwien.ac.at

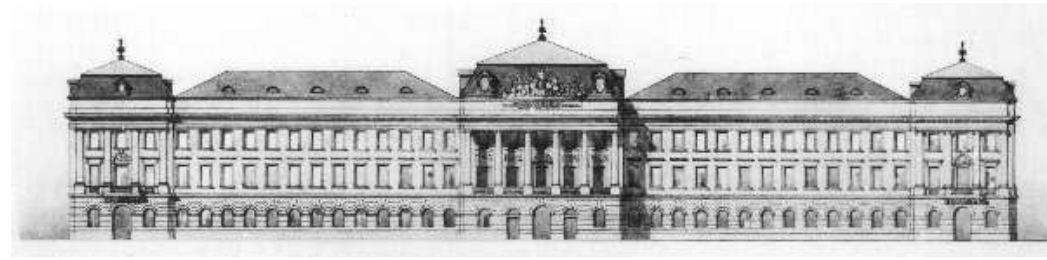

INSTITUT FÜR INFORMATIONSSYSTEME

AbTEILUNG DATENBANKEN UND ARTIFICIAL INTELLIGENCE

\section{Fast Counting with Bounded Treewidth}

\author{
DBAI-TR-2008-61
}

$\begin{array}{ccc}\text { Michael Jakl } & \begin{array}{c}\text { Reinhard Pichler } \\ \text { Stefan Woltran }\end{array} & \text { Stefan Rümmele }\end{array}$

DBAI TECHNICAL REPORT

2008

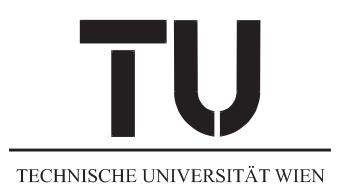




\author{
DBAI TECHNICAL REPORT \\ DBAI TECHNICAL REPORT DBAI-TR-2008-61, 2008
}

\title{
Fast Counting with Bounded Treewidth
}

\section{Michael Jakl Reinhard Pichler Stefan Rümmele Stefan Woltran ${ }^{1}$}

\begin{abstract}
Many intractable problems have been shown to become tractable if the treewidth of the underlying structure is bounded by a constant. An important tool for deriving such results is Courcelle's Theorem, which states that all properties defined by Monadic-Second Order (MSO) sentences are fixed-parameter tractable with respect to the treewidth. Arnborg et al. extended this result to counting problems defined via MSO properties. However, the MSO description of a problem is of course not an algorithm. Consequently, proving the fixed-parameter tractability of some problem via Courcelle's Theorem can be considered as the starting point rather than the endpoint of the search for an efficient algorithm. Gottlob et al. have recently presented a new approach via monadic datalog to actually devise efficient algorithms for decision problems whose tractability follows from Courcelle's Theorem. In this paper, we extend this approach and apply it to some fundamental counting problems in logic an artificial intelligence.
\end{abstract}

\footnotetext{
${ }^{1}$ Institute for Information Systems 184/2, Technische Universität Wien, Favoritenstrasse 9-11, 1040 Vienna, Austria. E-mail: \{jakl | pichler | ruemmele | woltran $\}$ @dbai.tuwien.ac.at

Acknowledgements: The authors acknowledge support by the Austrian Science Fund (FWF) grant P20704-N18.

This is an extended version of a paper published in the Proceedings of the 15th International Conference on Logic for Programming, Artificial Intelligence, and Reasoning (LPAR'08).

Copyright (c) 2009 by the authors
} 


\section{Introduction}

Many problems which are, in general, intractable, have been shown to become tractable if the treewidth of the underlying structure is bounded by a constant. An important tool for deriving such results is Courcelle's Theorem [1]. It states that any property of finite structures, which is expressible by a Monadic Second Order (MSO) sentence, can be decided in linear time (data complexity) if the structures under consideration have bounded treewidth. Courcelle's Theorem has been successfully applied to derive tractability results in a great variety of fields. Recently, also its applicability to AI has been underlined by showing that many fundamental problems in the area of nonmonotonic reasoning and knowledge representation can be encoded as MSO sentences [2]. In [3], it was shown that the fixed-parameter tractability (FPT) via Courcelle's Theorem can be extended to counting problems defined via MSO properties.

Clearly, the MSO description of a problem is not an algorithm. Previous methods for constructing concrete algorithms from an MSO description [3,4] first transform the MSO evaluation problem into a tree language recognition problem, which is then solved via a finite tree automaton (FTA). However, this approach has turned out to be only of theoretical value, since even very simple MSO formulae quickly lead to a "state explosion" of the FTA (see [5]). Consequently, it was already stated in [6] that the algorithms derived via Courcelle's Theorem are "useless for practical applications" and that the main benefit of Courcelle's Theorem is in providing "a simple way to recognize a property as being linear time computable". Of course, this also applies to the extension of Courcelle's Theorem to counting problems according to [3]. In other words, proving the FPT of some problem by showing that it is MSO expressible is the starting point rather than the end point of the search for an efficient algorithm.

Recently, an alternative method to tackle this class of fixed-parameter tractable problems via monadic datalog has been proposed in [7]. In particular, it has been shown that if some property of finite structures is expressible in MSO then it can also be expressed by means of a monadic datalog program over the structure plus the tree decomposition. The monadic datalog approach has been applied to problems from different areas $[7,8]$ including propositional satisfiability (SAT) and abduction. In this paper, we show that the monadic datalog approach can be extended in such a way that it also provides concrete algorithms for some fundamental counting problems.

Results. We present new algorithms for the following problems: \#S AT - the problem of counting all models of a propositional formula (without restriction, this is a classical \#P-complete problem); \#CIRCUMSCRIPTION - the problem of counting the (subset) minimal models of a propositional formula (this problem was, apart from the generic $\# \Pi_{1} \mathrm{SAT}$-problem, one of the first problems to be shown \#NP-complete [9]); and \#HORN-ABDUCTION - the problem of counting the solutions of a propositional abduction problem where the underlying theory is given by a set of Horn clauses. The \#P-completeness of this problem has been recently shown in [10]. Finally, we also report on experimental evaluations of the \#SAT algorithm. In particular, we compare a dedicated implementation (where datalog serves as a "specification") with direct realizations of the datalog approach on top of the DLV-system [11]. Our experiments underline that our approach of counting indeed yields the expected fixed-parameter tractability and that - in great contrast to the MSO-to-FTA approach - there are no "hidden constants" in the runtime behavior to render these algorithms useless. 
Related Work. As mentioned above, counting problems defined via MSO properties were shown in [3] to be FPT w.r.t. the treewidth of the input structures. In [12], this FPT result was extended to graphs with bounded clique-width. An algorithm for solving \#SAT and \#GENS AT in case of bounded treewidth or clique-width of the primal or incidence graph was presented in [13]. Moreover, it is sketched how this approach based on recursive splitting can be extended to other \#P-complete problems. In [14], new \#S ATalgorithms based on dynamic programming were presented for bounded treewidth of several graphs related to a propositional formula in CNF, namely the primal graph, dual graph, and incidence graph. Our notion of treewidth of a CNF-formula (see Section 2) corresponds to the treewidth of the incidence graph.

\section{Preliminaries}

Finite Structures and Treewidth. Let $\tau=\left\{R_{1}, \ldots, R_{K}\right\}$ be a set of predicate symbols. A finite structure $\mathcal{A}$ over $\tau$ (a $\tau$-structure, for short) is given by a finite domain $A=\operatorname{dom}(\mathcal{A})$ and relations $R_{i}^{\mathcal{A}} \subseteq A^{\alpha}$, where $\alpha$ is the arity of $R_{i} \in \tau$. A tree decomposition $\mathcal{T}$ of a $\tau$-structure $\mathcal{A}$ is a pair $\left\langle T,\left(A_{t}\right)_{t \in T}\right\rangle$ where $T$ is a tree and each $A_{t}$ is a subset of $A$, s.t. the following properties hold: (1) Every $a \in A$ is contained in some $A_{t}$. (2) For every $R_{i} \in \tau$ and every tuple $\left(a_{1}, \ldots, a_{\alpha}\right) \in R_{i}^{\mathcal{A}}$, there exists a node $t \in T$ with $\left\{a_{1}, \ldots, a_{\alpha}\right\} \subseteq A_{t}$. (3) For every $a \in A,\left\{t \mid a \in A_{t}\right\}$ induces a subtree of $T$.

The sets $A_{t}$ are called the bags of $\mathcal{T}$. The width of a tree decomposition $\left\langle T,\left(A_{t}\right)_{t \in T}\right\rangle$ is defined as $\max \left\{\left|A_{t}\right| \mid t \in T\right\}-1$. The treewidth of $\mathcal{A}$ is the minimal width of all tree decompositions of $\mathcal{A}$. It is denoted as $t w(\mathcal{A})$. For given $w \geq 1$, it can be decided in linear time if some structure has treewidth $\leq w$. Moreover, in case of a positive answer, a tree decomposition of width $w$ can be computed in linear time [15].

Example 1 ([2]). We can represent propositional formulae in CNF as finite structures over the alphabet $\tau=\{\operatorname{cl}(),. \operatorname{var}(),. \operatorname{pos}(.,),. n e g(.,)$.$\} where \operatorname{cl}(z)($ resp. $\operatorname{var}(z))$ means that $z$ is a clause (resp. a variable) and $\operatorname{pos}(x, c)$ (resp. neg $(x, c)$ ) means that $x$ occurs unnegated (resp. negated) in the clause $c$. For instance, the formula $\varphi=\left(x_{1} \vee\right.$ $\left.\neg x_{2} \vee x_{3}\right) \wedge\left(\neg x_{1} \vee x_{4} \vee \neg x_{5}\right) \wedge\left(x_{2} \vee \neg x_{4} \vee x_{6}\right)$ corresponds to the structure $\mathcal{A}$ given by the set of ground atoms $\left\{\operatorname{var}\left(x_{1}\right), \operatorname{var}\left(x_{2}\right), \operatorname{var}\left(x_{3}\right), \operatorname{var}\left(x_{4}\right), \operatorname{var}\left(x_{5}\right), \operatorname{var}\left(x_{6}\right)\right.$, cl $\left(c_{1}\right), \operatorname{cl}\left(c_{2}\right), \operatorname{cl}\left(c_{3}\right), \operatorname{pos}\left(x_{1}, c_{1}\right), \operatorname{pos}\left(x_{3}, c_{1}\right), \operatorname{pos}\left(x_{4}, c_{2}\right), \operatorname{pos}\left(x_{2}, c_{3}\right), \operatorname{pos}\left(x_{6}, c_{3}\right)$, $\left.n e g\left(x_{2}, c_{1}\right), \operatorname{neg}\left(x_{1}, c_{2}\right), \operatorname{neg}\left(x_{5}, c_{2}\right), \operatorname{neg}\left(x_{4}, c_{3}\right)\right\}$. Two tree decompositions $\mathcal{T}_{1}$ and $\mathcal{T}_{2}$ of $\mathcal{A}$ are given in Figure 1. Note that the maximal size of the bags is 3 in both decompositions. Hence, the treewidth is $\leq 2$. On the other hand, it can be shown that these tree decompositions are optimal in the sense that we have $t w(\varphi)=t w(\mathcal{A})=2 . \diamond$

In [7], it was shown that the following form of normalized tree decompositions can be obtained in linear time: (1) All bags contain either $w$ or $w+1$ pairwise distinct elements. W.l.o.g., we may assume that the domain contains at least $w$ elements. (2) Every internal node $t \in T$ has either 1 or 2 child nodes. (3) If a node $t$ has one child node $t^{\prime}$, then the bag $A_{t}$ is obtained from $A_{t^{\prime}}$ either by removing one element or by introducing a new element. (4) If a node $t$ has two child nodes then these child nodes have identical bags as $t$. In this case, we call $t$ a branch node. In this paper, we only deal with finite structures representing propositional formulae in CNF (possibly Horn). Hence, the domain elements are either variables or clauses. Consequently, in case (3), 


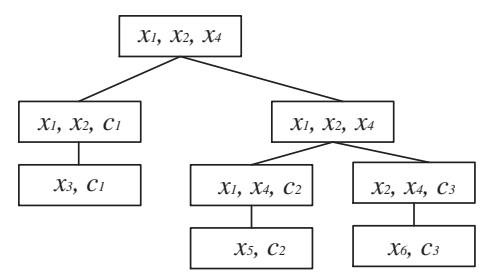

(a) tree decomposition $\mathcal{T}_{1}$

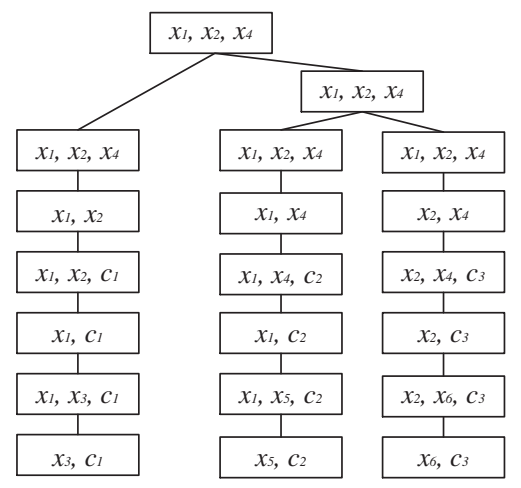

(b) tree decomposition $\mathcal{T}_{2}$

Fig. 1. Tree decompositions of formula $\varphi$ of Example 1.

we call a node $t$ in the tree decomposition a variable removal node, a clause removal node, a variable introduction node, or a clause introduction node, respectively.

The tree decomposition $\mathcal{T}_{2}$ in Figure 1 is normalized in this sense.

MSO and Monadic Datalog. MSO extends First Order logic (FO) by the use of set variables (denoted by upper case letters), which range over sets of domain elements. In contrast, the individual variables (denoted by lower case letters) range over single domain elements. An MSO formula $\varphi(x)$ with exactly one free individual variable is called a unary query. Datalog programs are function-free logic programs. The (minimal-model) semantics can be defined as the least fixpoint (lfp) of applying the immediate consequence operator. Predicates occurring only in the body of rules are called extensional. Predicates occurring also in the head of some rule are called intensional.

Let $\mathcal{A}$ be a $\tau$-structure of treewidth $w \geq 1$. Then we define the extended signature $\tau_{t d}=\tau \cup\left\{\right.$ root, leaf, child $_{1}$, child $_{2}$, bag $\}$, where the unary predicates root and leaf as well as the binary predicates child $_{1}$ and child $_{2}$ are used to represent the tree of a tree decomposition (of width $w$ ) in the obvious way. Finally, predicate $b a g$ has arity $k+2$ with $k \leq w$, where $\operatorname{bag}\left(t, a_{0}, \ldots, a_{k}\right)$ means that the bag at node $t$ is $\left(a_{0}, \ldots, a_{k}\right)$.

In [7], the following connection between unary MSO queries over structures with bounded treewidth and monadic datalog was established:

Theorem 1. Let $\tau$ and $w \geq 1$ be arbitrary but fixed. Every MSO-definable unary query over $\tau$-structures of treewidth $w$ is also definable by a monadic datalog program over $\tau_{t d}$. Moreover, the resulting program can be evaluated in linear time w.r.t. the size of the original $\tau$-structure.

\section{Counting all models}

We start our investigation of counting problems with the \#SAT problem, i.e.: given a clause set $\mathcal{C}$ over variables $V$, count the number of all models $J \subseteq V$ of $\mathcal{C}$ (We identify an assignment with the set of atoms that are true in it). Suppose that an instance of \#SAT is given as a $\tau_{t d}$-structure with $\tau_{t d}=\left\{c l\right.$,var,pos, neg, root, leaf, child $_{1}$, child $\left._{2}, b a g\right\}$, encoding a clause set together with a tree decomposition $\mathcal{T}$ of width $w$ (as explained Example 1). An extended datalog program for \#S AT is displayed in Figure 2. 


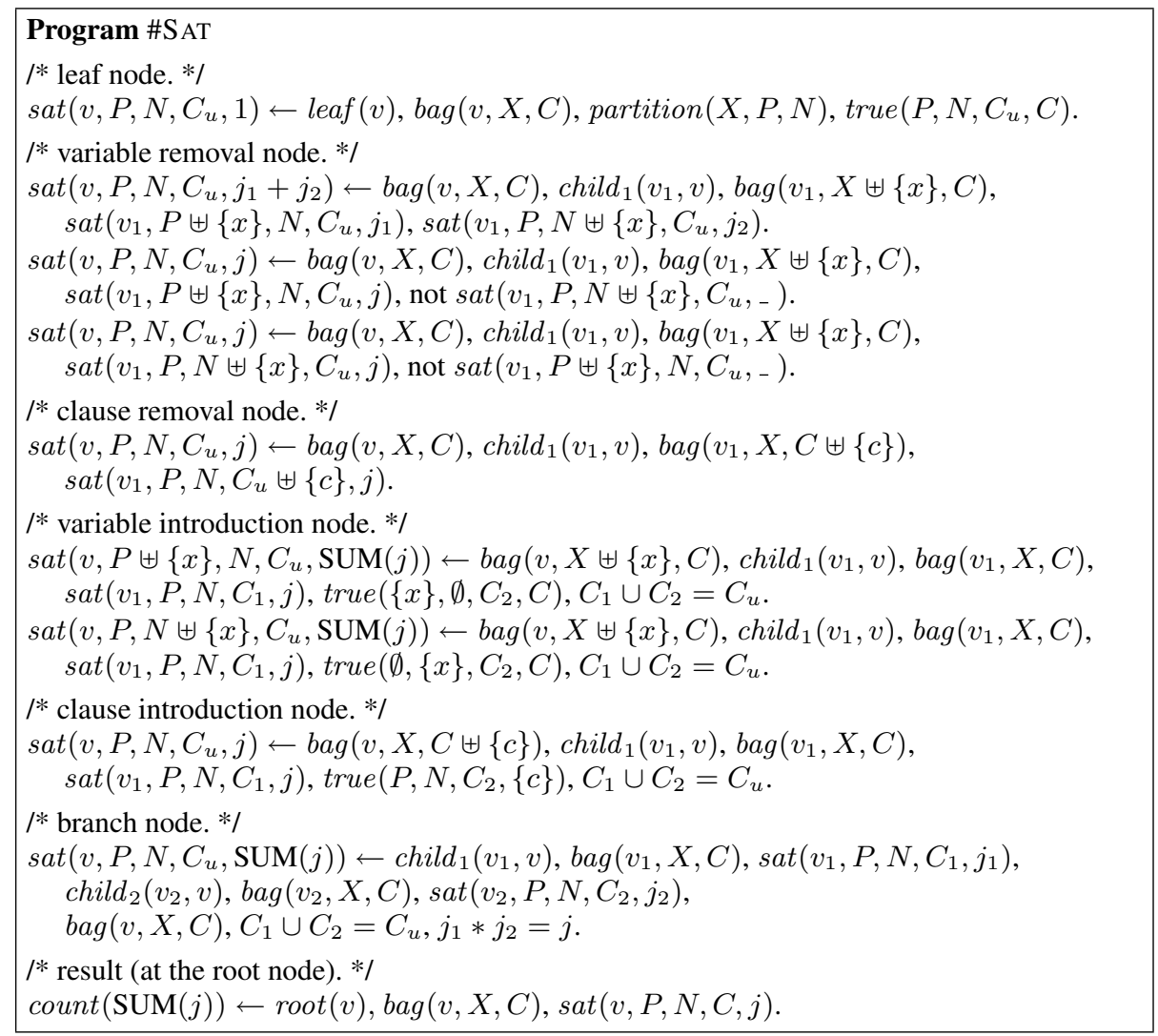

Fig. 2. \#SAT program.

In this program, we adhere to the following notational conventions: Lower case letters $v, c, x$, and $j$ (possibly with subscripts) are used as datalog variables for a single node in $\mathcal{T}$, for a single clause, for a single propositional variable, or for an integer number, respectively. Upper case letters are used as datalog variables denoting sets of variables (in the case of $X, P, N$ ) or sets of clauses (in the case of $C$ ). In particular, for the sake of readability, we present the extensional predicate $\operatorname{bag}$ in the form $\operatorname{bag}(v, X, C)$, where $X$ (resp. $C$ ) denotes the set of variables (resp. clauses) in the bag at node $v$ in $\mathcal{T}$. Note that all these sets are not sets in the general sense, since their cardinality is restricted by the maximal size $w+1$ of the bags, where $w$ is a fixed constant. Indeed, we ultimately feed these sets to the datalog system DLV in the form of individual arguments of appropriate variants of the predicates involved, see Section 6.

We are also using non-datalog expressions involving the $\cup$ - and $\uplus$-operator for ordinary resp. disjoint union. They could be easily replaced by "proper" datalog expressions, e.g., $C_{1} \cup C_{2}=C_{u}$ can of course be replaced by union $\left(C_{1}, C_{2}, C_{u}\right)$. Moreover, we need arithmetic expressions $j_{1}+j_{2}$ and $j_{1} * j_{2}$ as well as the SUM-operator for the counting. The SUM-operator occurs as the expression $\operatorname{SUM}(j)$ in the rule heads 
only. Its semantics is like the SUM aggregate function in ordinary SQL, where we first apply a GROUP BY over all remaining head variables to the result of evaluating the conjunctive query in the body of the rule.

For the discussion of the \#SAT program below, it is convenient to introduce the following notation: Let $\mathcal{C}$ denote the input clause set with variables in $V$ and tree decomposition $\mathcal{T}$. For any node $v$ in $\mathcal{T}$, we write $\mathcal{T}_{v}$ to denote the subtree of $\mathcal{T}$ rooted at $v$. By $C l(v)$ we denote the clauses in the bag of $v$ while $C l\left(\mathcal{T}_{v}\right)$ denotes the clauses that occur in any bag in $\mathcal{T}_{v}$. Analogously, we write $\operatorname{Var}(v)$ and $\operatorname{Var}\left(\mathcal{T}_{v}\right)$ as a short-hand for the variables occurring in the bag of $v$ respectively in any bag in $\mathcal{T}_{v}$. Finally, the restriction of a clause $c$ to the variables in some set $U \subseteq V$ will be denoted by $\left.c\right|_{U}$.

The \#SAT program contains four intensional predicates sat, true, partition, and count. The crucial predicate is $\operatorname{sat}(v, P, N, C, j)$ with the following intended meaning: $v$ denotes a node in $\mathcal{T}$. $P$ and $N$ form a partition of $\operatorname{Var}(v)$ representing a truth assignment on $\operatorname{Var}(v)$, s.t. all variables in $P$ are true and all variables in $N$ are false. $C$ denotes a subset of $C l(v)$ and $j$ denotes a positive integer. For arbitrary values of $v, P, N, C$, we define the following set of truth assignments:

$$
\begin{aligned}
S(v, P, N, C)=\{J \mid & J \text { is an extension of }(P, N) \text { to } \operatorname{Var}\left(\mathcal{T}_{v}\right), \\
& \text { for each } c \in\left(C l\left(\mathcal{T}_{v}\right) \backslash C l(v)\right) \cup C, c \text { is true in } J, \\
& \text { for each } \left.c \in C l(v) \backslash C,\left.c\right|_{\operatorname{Var}\left(\mathcal{T}_{v}\right)} \text { is false in } J .\right\}
\end{aligned}
$$

We can now characterize the least fixpoint (lfp) of the \#SAT program as follows.

Property A. If $S(v, P, N, C)=\emptyset$ then no atom $\operatorname{sat}\left(v, P, N, C,{ }_{-}\right)$is in the lfp of \#S AT. If $S(v, P, N, C) \neq \emptyset$ then the following equivalence holds: $\operatorname{sat}(v, P, N, C, j)$ is in the lfp of \#S AT iff $|S(v, P, N, C)|=j$.

This property implies that, for any given values $v, P, N, C$, we can derive at most one fact $\operatorname{sat}(v, P, N, C, j)$. The main task of the program is the computation of all facts $\operatorname{sat}(v, P, N, C, j)$ by means of a bottom-up traversal of the tree decomposition $\mathcal{T}$. Indeed, all the rules only allow us to derive sat-facts for some node $v$ in $\mathcal{T}$ from sat-facts at the child node(s) of $v$. Consequently, on the ground level, the program contains only stratified negation, since the not-operator in the rules of variable removal nodes is only applied to $s a t$-facts of the child node $v_{1}$ of $v$.

The other predicates have the following meaning: $\operatorname{true}\left(P, N, C_{u}, C\right)$ means that $C_{u}$ contains precisely those clauses from $C$ which are true in the (partial) assignment given by $(P, N)$. We do not specify the implementation of this predicate here. It can be easily achieved via the extensional predicates pos and neg. A fact partition $(X, P, N)$ expresses that $(P, N)$ is a partition of $X$. The predicate count holds the final result. The datalog program in Figure 2 solves the \#S AT problem in the following way.

Theorem 2. Let $\mathcal{C}$ be an instance of \#S AT, encoded by a $\tau_{t d}$-structure $\mathcal{A}_{t d}$. Then, count $(j)$ with $j \geq 1$ is in the lfp of the \#SAT-program evaluated on $\mathcal{A}_{t d}$ iff $\mathcal{C}$ is satisfiable and has exactly $j$ models. Moreover, both the construction of the $\tau_{t d}$-structure $\mathcal{A}_{t d}$ and the evaluation of the program take time $\mathcal{O}(f(t w(\mathcal{C})) *\|\mathcal{C}\|)$ for some function $f$, if we assume constant runtime for the arithmetic operations.

Proof. Suppose that the predicate sat indeed fulfills Property A, which can be proved by structural induction on $\mathcal{T}$. The case distinction over all possible kinds of nodes is 
rather straightforward - the only non-trivial case being the case of branch nodes. A detailed proof is given in Appendix A.

Now consider the root node $v$ of the tree decomposition $\mathcal{T}$ with $\operatorname{bag}(v, X, C)$. A fact $\operatorname{sat}(v, P, N, C, j)$ in the lfp means that the assignment $(P, N)$ on the variables $X$ has exactly $j$ extensions to all variables, s.t. all clauses in $\mathcal{C}$ are true. But then, by the semantics of the SUM-operator explained above, the rule with head $\operatorname{count}(\operatorname{SUM}(j))$ indeed means that a fact count $\left(j^{\prime}\right)$ with $j^{\prime} \geq 1$ is in the lfp iff $j^{\prime}$ is the number of assignments that satisfy all clauses in $\mathcal{C}$, i.e., $j^{\prime}$ is the sum of $j$ over all possible partitions $(P, N)$ of $X$, s.t. $\operatorname{sat}(v, P, N, C, j)$ is in the lfp. We are thus using that the root $v$ of $\mathcal{T}$ is unique and the values of $X$ and $C$ in the bag at $v$ are uniquely determined by $v$. Moreover, for every pair of $(P, N)$ (together with $C$, which is fixed for $v$ ), the value of $j$ is also uniquely determined.

The linear time data complexity is due to the fact that our \#SAT program is essentially a succinct representation of a monadic datalog program extended by a counter $j$. For instance, in the atom $\operatorname{sat}(v, P, N, C, j)$, the sets $P, N$, and $C$ are subsets of bounded size of the bag of $v$. Hence, each combination $P, N, C$ could be represented by sets $r, s, t \subseteq\{0, \ldots, w\}$ referring to indices of elements in the bag of $v$. Recall that $w$ is a fixed constant. Hence, $\operatorname{sat}(v, P, N, C, j)$ is simply a succinct representation of constantly many predicates of the form $\operatorname{sat}_{r, s, t}(v, j)$. Hence, without the counter $j$, the linear time bound is implicit in Theorem 1. Moreover, $j$ is uniquely determined for every combination of $v, P, N, C$, and the concrete value of $j$ is computed by simple addition and multiplication of the corresponding values in sat-facts at the child node(s) of $v$. Hence, maintaining this additional argument $j$ does not destroy the linearity.

\section{Counting the minimal models}

We now extend the \#SAT program in order to solve the \#CIRCUMSCRIPTION problem, i.e.: given a propositional formula $\varphi$, count the number of minimal models of $\varphi$. The goal of the program in Figure 3 and 4 is, on the one hand, to keep track of all models of a formula $\varphi$ given by the input $\tau_{t d}$-structure. This is done by the sat-predicate which works essentially as in the \#SAT program. However, at the end of the day, we may only count the minimal models. Our \#CIRCUMSCRIPTION program therefore also contains an unsat-predicate, which is used to propagate "unsat"-conditions in the sense that some model $J$ is minimal only if all strictly smaller assignments $J^{\prime} \subset J$ do not satisfy $\varphi$. Recall that we identify an assignment with the set of atoms that are true in it.

A complication which our program has to overcome is that we have to keep track which unsat-conditions refer to which sat-condition. Thus the sat-predicate has an index $i \in\{0,1,2, \ldots\}$ as additional argument. The first four arguments $v, i, P, N$ allow us to associate each unsat-fact with the correct sat-fact. The sat-and unsat-predicates have the following meaning: Let $v$ denote a node in the tree decomposition $\mathcal{T}$. Let the sets $P$ and $N$ (resp. $P^{\prime}$ and $N^{\prime}$ ) denote a partition of $\operatorname{Var}(v)$ representing a truth assignment on $\operatorname{Var}(v)$, s.t. all variables in $P$ (resp. in $P^{\prime}$ ) are true and all variables in $N$ (resp. in $N^{\prime}$ ) are false. Let $C$ and $C^{\prime}$ denote subsets of $C l(v)$. Furthermore let $i \in\{0,1,2, \ldots\}$ be an index used to distinguish different extensions of a truth assignment and let $j$ be a positive integer used for counting extensions of a truth assignment. Moreover, let the set $S(v, P, N, C)$ of truth assignments be defined as in Section 3 . Then, occurrences of the ground facts $\operatorname{sat}(v, i, P, N, C, j)$ and unsat $\left(v, i, P, N, P^{\prime}, N^{\prime}, C^{\prime}\right)$ in the least fixpoint (lfp) of \#CIRCUMSCRIPTION are determined as follows: 


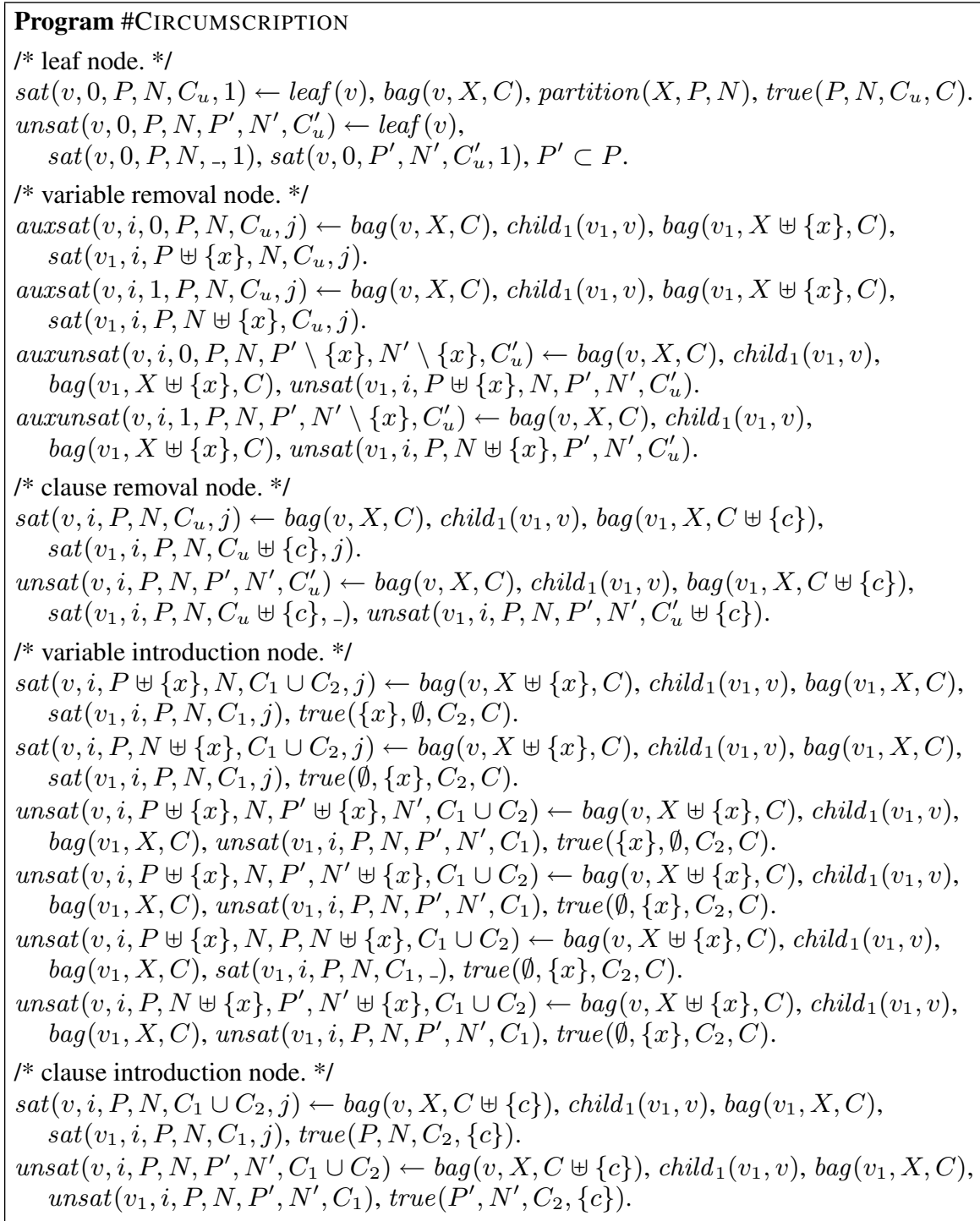

Fig. 3. \#CIRCUMSCRIPTION program.

Property B. There exists an atom $\operatorname{sat}\left(v,,_{-}, P, N, C,_{-}\right)$in the lfp of the \#CIRCUMSCRIPTION program iff $S(v, P, N, C) \neq \emptyset$. Moreover, a fact unsat $\left(v, i, P, N,_{-},,_{-},,_{-},-\right)$is in the lfp only if also a fact $\operatorname{sat}\left(v, i, P, N,_{-},{ }_{-}\right)$is. Finally, if $S(v, P, N, C) \neq \emptyset$ then there exists a partition $\left\{S_{i_{1}}, \ldots, S_{i_{n}}\right\}$ with $n \geq 1$ of $S(v, P, N, C)$ which fulfills the following conditions:

1. A fact $\operatorname{sat}\left(v, i, P, N, C{ }_{-}\right)$is contained in the lfp iff $i \in\left\{i_{1}, \ldots, i_{n}\right\}$. 


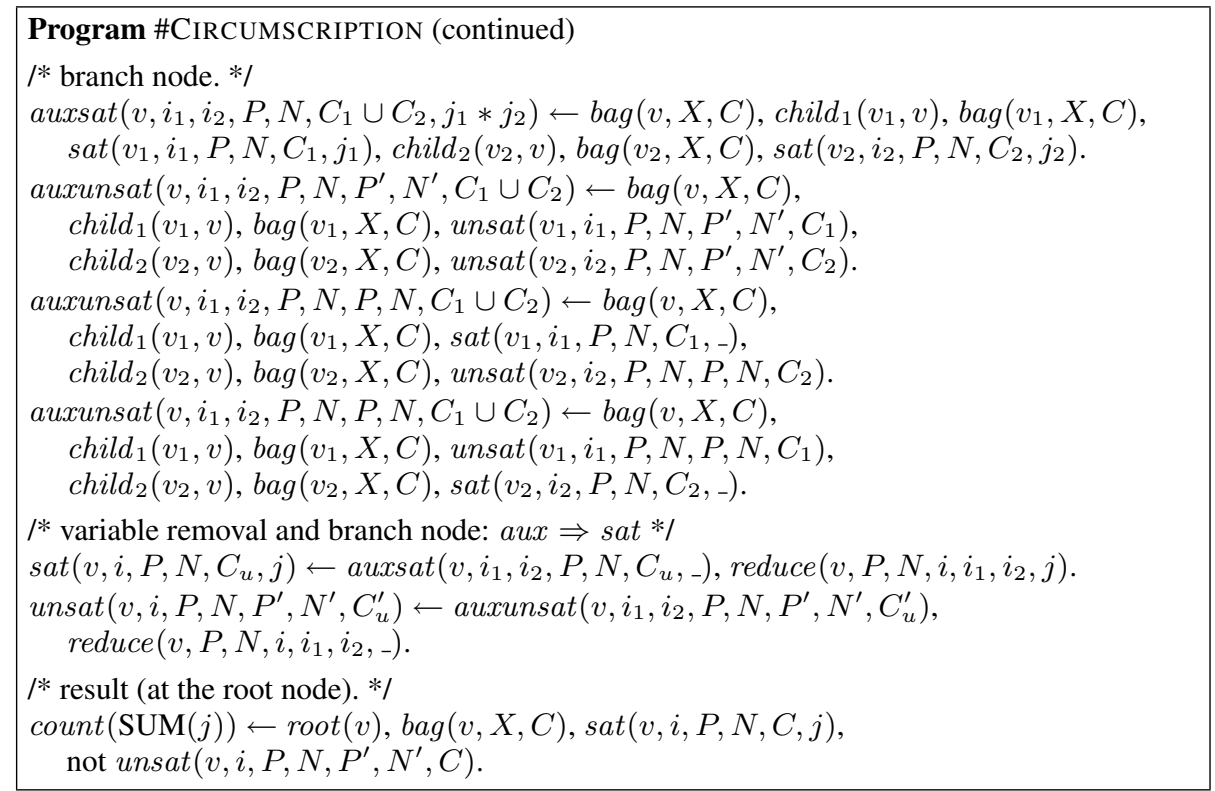

Fig. 4. \#CIRCUMSCRIPTION program.

2. The fact $\operatorname{sat}(v, i, P, N, C, j)$ is contained in the lfp iff $\left|S_{i}\right|=j$.

3. For every partition $\left(P^{\prime}, N^{\prime}\right)$ of $\operatorname{Var}(v)$ and every subset $C^{\prime} \subseteq C l(v)$, the following two equivalences hold:

The fact unsat $\left(v, i, P, N, P^{\prime}, N^{\prime}, C^{\prime}\right)$ is contained in the lfp $\Leftrightarrow$ there exists a $J \in S_{i}$ and an assignment $J^{\prime} \subset J$, s.t. $J^{\prime} \in S\left(v, P^{\prime}, N^{\prime}, C^{\prime}\right) \Leftrightarrow$ for all $J \in S_{i}$ there exists an assignment $J^{\prime} \subset J$, s.t. $J^{\prime} \in S\left(v, P^{\prime}, N^{\prime}, C^{\prime}\right)$.

Condition 2 above implies that, for any values $v, i, P, N, C$, there is at most one fact $\operatorname{sat}\left(v, i, P, N, C,{ }_{-}\right)$in the lfp. Condition 3 ensures that, at the root node $v$ of $\mathcal{T}$, either all $j$ models described by a fact $\operatorname{sat}(v, i, P, N, C, j)$ are minimal or none of them is.

The predicates true and partition have the same meaning as in the \#SAT program. In addition, we have the predicates auxsat, auxunsat, and reduce with the following meaning: Recall that the index $i$ in $\operatorname{sat}\left(v, i, P, N, C,{ }_{-}\right)$is used to keep different assignments $J \in S(v, P, N, C)$ apart. Of course, in principle, there can be exponentially many such $J$. Nonetheless, the predicates auxsat, auxunsat, and reduce guarantee the fixed-parameter tractability in the following way. In the first place, we compute facts auxsat $\left(v, i_{1}, i_{2}, P, N, C,{ }_{-}\right)$and auxunsat $\left(v, i_{1}, i_{2}, P, N, P^{\prime}, N^{\prime}, C^{\prime}\right)$, where we use pairs of indices $\left(i_{1}, i_{2}\right)$ rather than a single index $i$ to associate the auxunsatfacts with the correct auxsat-fact. Now suppose that for two distinct pairs $\left(i_{1}, i_{2}\right)$ and $\left(i_{1}^{\prime}, i_{2}^{\prime}\right)$ a fact $\operatorname{auxsat}\left(v, i_{1}, i_{2}, P, N, C,{ }_{-}\right)$and $\operatorname{auxsat}\left(v, i_{1}^{\prime}, i_{2}^{\prime}, P, N, C,{ }_{-}\right)$exists in the lfp and, moreover, the auxunsat-facts for $\left(v, i_{1}, i_{2}, P, N\right)$ and $\left(v, i_{1}^{\prime}, i_{2}^{\prime}, P, N\right)$ are the same, i.e., for indices $i, j$, let $\operatorname{Val}(v, i, j, P, N)=\left\{\left(P^{\prime}, N^{\prime}, C^{\prime}\right) \mid\right.$ there exists a fact auxunsat $\left(v, i, j, P, N, P^{\prime}, N^{\prime}, C^{\prime}\right)$ in the lfp $\}$. Then $\operatorname{Val}\left(v, i_{1}, i_{2}, P, N\right)=\operatorname{Val}\left(v, i_{1}^{\prime}\right.$, $\left.i_{2}^{\prime}, P, N\right)$ holds. Intuitively, this means that the pairs of indices $\left(i_{1}, i_{2}\right)$ and $\left(i_{1}^{\prime}, i_{2}^{\prime}\right)$ 
are not distinguishable by the sat- and unsat-conditions for this particular combination of $(v, P, N)$. The purpose of the reduce-predicate is, in such a situation, to contract $\left(i_{1}, i_{2}\right)$ and $\left(i_{1}^{\prime}, i_{2}^{\prime}\right)$ to a single index $i$ and to take care of the actual counting and summation. More precisely, a fact reduce $\left(v, P, N, i, i_{1}, i_{2}, j\right)$ means that the pair of indices $\left(i_{1}, i_{2}\right)$ is mapped to the single index $i$ and that $j$ is the sum of all $j^{\prime}$ in facts auxsat $\left(v, i_{1}^{\prime}, i_{2}^{\prime}, P, N, C, j^{\prime}\right)$, s.t. $\left(i_{1}^{\prime}, i_{2}^{\prime}\right)$ is mapped to $i$. In principle, the reducepredicate predicate can be realized in datalog (see Appendix B). However, in the long run, an efficient implementation via hash tables inside the datalog processor is clearly preferable. The datalog program in Figure 3 and 4 solves the \#CIRCUMSCRIPTION problem in the following way:

Theorem 3. Let $\mathcal{C}$ be an instance of \#CIRCUMSCRIPTION, encoded by a $\tau_{t d-\text { structure }}$ $\mathcal{A}_{t d}$. Then, count $(j)$ with $j \geq 1$ is in the lfp of the \#CIRCUMSCRIPTION-program evaluated on $\mathcal{A}_{t d}$ iff $\mathcal{C}$ is satisfiable and has exactly $j$ (subset) minimal models. Moreover, both the construction of the $\tau_{t d}$-structure $\mathcal{A}_{t d}$ and the evaluation of the program take time $\mathcal{O}(f(t w(\mathcal{C})) *\|\mathcal{C}\|)$ for some function $f$, if we assume constant runtime for the arithmetic operations.

Proof. The proof is based on essentially the same ideas as the proof of Theorem 2 . In particular, the correctness follows easily as soon as the correctness of Property B is established, which can be done by structural induction (see Appendix C). The linear time bound is again shown via Theorem 1 and the fact that the arithmetic operations required for the counting do not destroy the linear time data complexity.

\section{Horn abduction}

Abduction is an important method in artificial intelligence and, in particular, in diagnosis. A propositional abduction problem (PAP) is given by a tuple $\mathcal{P}=\langle V, H, M, \mathcal{C}\rangle$, where $V$ is a finite set of variables, $H \subseteq V$ is the set of hypotheses, $M \subseteq V$ is the set of manifestations and $\mathcal{C}$ is a consistent theory in the form of a propositional clause set. A set $\mathcal{S} \subseteq H$ is a solution to $\mathcal{P}$ if $\mathcal{C} \cup \mathcal{S}$ is consistent and $\mathcal{C} \cup \mathcal{S} \models M$ holds.

In [8], the decision problem (i.e., does a given PAP have a solution) of propositional abduction with bounded treewidth was considered. In order to illustrate the wide applicability of the datalog approach, we concentrate on the special case of \#HORNABDUCTION, i.e., given a PAP $\mathcal{P}$ whose theory is a set of Horn clauses, count the number of solutions $\mathcal{S}$ of $\mathcal{P}$. The datalog program in Figure 5 has a significantly different flavour than the ones in the previous sections and can be considered as prototypical for rule-based problems.

Before we explain this program, we introduce some useful terminology and conventions: In general, Horn clauses are either rules, facts, or goals. For our purposes, it is convenient to consider every clause $r$ of $\mathcal{C}$ as a rule consisting of a head (denoted as $h e a d(r)$ ) and a body (denoted as body $(r)$ ). Goals of the form $\neg p_{1} \vee \cdots \vee \neg p_{k}$ are thus considered as rules of the form $p_{1} \wedge \cdots \wedge p_{k} \rightarrow \perp$ and a fact $q$ in $\mathcal{C}$ is considered as a rule of the form $\rightarrow q$ with an empty body. A PAP is represented by a $\tau$-structure with $\tau=\{c l, v a r, n e g$, pos, hyp, man $\}$, where the predicates hyp and man indicate that some variable $a$ is a hypothesis (i.e., $\operatorname{hyp}(a)$ ) or a manifestation (i.e., $\operatorname{man}(a)$ ). By the above consideration, $\operatorname{var}(\perp)$ is now also fulfilled. Moreover, $\operatorname{neg}(a, r)($ resp. $\operatorname{pos}(a, r))$ 


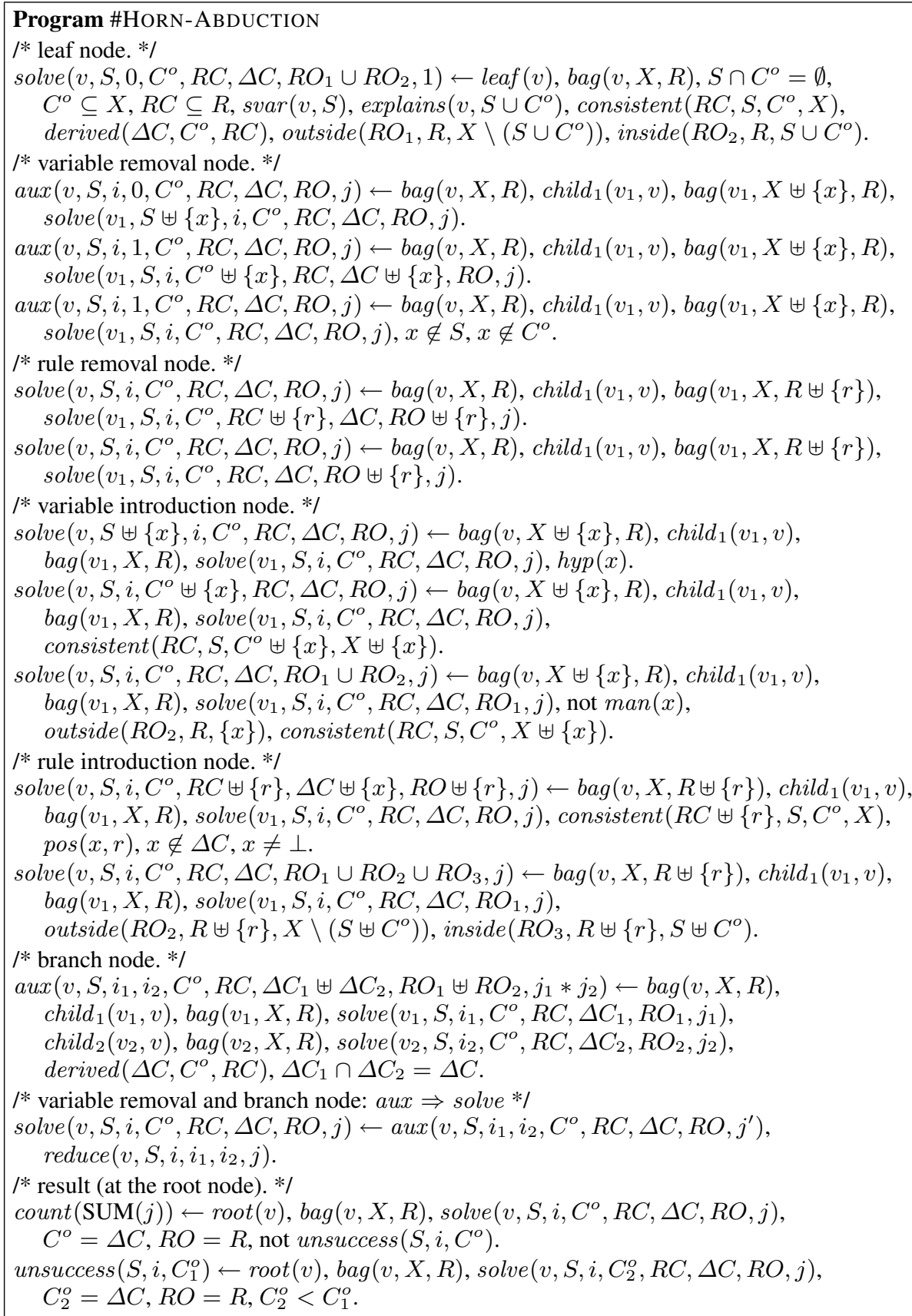

Fig. 5. \#HORN-ABDUCTION program. 
means that $a$ occurs in the body of $r$ (resp. in the head of $r$ ). For the input tree decomposition, we assume that a bag containing some rule $r$ also contains the variable $a$ in the head of $r$. This will greatly simplify the presentation of our datalog program and can, in the worst-case, only double the width of the resulting decomposition.

For $\mathcal{S} \subseteq V \cup\{\perp\}$, we write $\mathcal{S}^{+}$to denote the closure of $\mathcal{S}$ w.r.t. the theory $\mathcal{C}$, i.e.: An element $q \in V \cup\{\perp\}$ is contained in $\mathcal{S}^{+}$iff either $q \in \mathcal{S}$ or there exists a "derivation sequence" of $q$ from $\mathcal{S}$ in $\mathcal{C}$ of the form $\mathcal{S} \rightarrow \mathcal{S} \cup\left\{q_{1}\right\} \rightarrow \mathcal{S} \cup\left\{q_{1}, q_{2}\right\} \rightarrow$ $\ldots \rightarrow \mathcal{S} \cup\left\{q_{1}, \ldots, q_{n}\right\}$, s.t. $q_{n}=q$ and for every $i \in\{1, \ldots, n\}$, there exists a rule $r_{i} \in \mathcal{C}$ with $\operatorname{body}\left(r_{i}\right) \subseteq \mathcal{S} \cup\left\{q_{1}, \ldots, q_{i-1}\right\}$ and head $\left(r_{i}\right)=q_{i}$. Hence, a subset $\mathcal{S} \subseteq H$ is a solution of the PAP $\mathcal{P}$ iff $\perp \notin \mathcal{S}^{+}$and $M \subseteq \mathcal{S}^{+}$. Our \#HoRN-ABDUCTION program searches for the number of solutions $\mathcal{S} \subseteq H$ by applying precisely this criterion. The predicate solve $\left(v, S, i, C^{o}, R C, \Delta C, R O, j\right)$, which is at the heart of the \#HORN-ABDUCTION program, has the following intended meaning: $v$ denotes a node in the tree decomposition $\mathcal{T}$. $S$ is the projection of a solution $\mathcal{S}$ onto $H y p(v)$ and $C^{o}$ is the projection of $\mathcal{S}^{+} \backslash \mathcal{S}$ onto $\operatorname{Var}(v)$. We consider $\mathcal{S}^{+} \backslash \mathcal{S}$ as well as $C^{o}$ as ordered (which is indicated by the superscript $o$ ) w.r.t. some derivation sequence of $\mathcal{S}^{+}$from $\mathcal{S}$. The arguments $R C, \Delta C$, and $R O$ are used to check that $C^{o}$ is indeed the projection of $\mathcal{S}^{+} \backslash \mathcal{S}$ onto $\operatorname{Var}(v)$. Informally, the arguments $R C$ and $\Delta C$ ensure that $C^{o}$ is not too big, while $R O$ ensures that $C^{o}$ is not too small. These tasks are accomplished as follows: $R C$ contains those rules in $v$ which are used in the above derivation sequence. Furthermore, the set $\Delta C$ contains those variables of $C^{o}$, for which we have already found the corresponding derivation rule. Of course, in the bottom-up traversal of the tree decomposition, every element of $C^{o}$ ultimately has to end up in $\Delta C$. On the other hand, $R O$ contains those rules $r$ in the bag of $v$ which do not constitute a contradiction with the closedness of $\mathcal{S}^{+}$, i.e., either the head of $r$ is contained in $\mathcal{S}^{+}$anyway or we have already encountered in $\operatorname{Var}\left(\mathcal{T}_{v}\right)$ a variable in $\operatorname{body}(r)$ which is not contained in $\mathcal{S}^{+}$. The last argument $j$ is used to count the number of different solutions.

In the program, we again use $\cup$ and $\uplus$ to denote ordinary union resp. disjoint union. By $C^{o} \uplus\{x\}$, we mean that $x$ is arbitrarily "inserted" into $C^{o}$, leaving the order of the remaining elements unchanged. Analogously to the \#CIRCUMSCRIPTION program, we need an index $i$ in order to distinguish between different derivation sequences leading to different orderings on the elements in $\mathcal{S}^{+} \backslash \mathcal{S}$. Moreover, we need an aux-predicate maintaining pairs of indices in case of variable removable and branch nodes. Moreover, we also need a reduce-predicate to contract $\operatorname{aux}$-facts $\operatorname{aux}\left(v, S, i_{1}, i_{2}, \ldots\right)$ and $\operatorname{aux}\left(v, S, i_{1}^{\prime}, i_{2}^{\prime}, \ldots\right)$ for partial solutions which are indistinguishable by the aux-facts in the lfp. The actual counting and summation is again done in the reduce-predicate (see Appendix D).

Formally, the correctness of the \#HORN-ABDUCTION program can be shown via the Property $\mathrm{C}$ defined below. Let $\operatorname{Hyp}(v), \operatorname{Man}(v), \operatorname{Hyp}\left(\mathcal{T}_{v}\right)$, and $\operatorname{Man}\left(\mathcal{T}_{v}\right)$ denote the restriction of $H$ and $M$ to the variables in the bag of $v$ or in any bag in $\mathcal{T}_{v}$, respectively. For arbitrary values of $v, S, C^{o}, R C, \Delta C$, and $R O$, we define the following set of extensions $\bar{S}$ of $S$ to $\operatorname{Hyp}\left(\mathcal{T}_{v}\right)$ :

$\operatorname{Sol}\left(v, S, C^{o}, R C, \Delta C, R O\right)=\left\{\bar{S} \mid S \subseteq \bar{S} \subseteq \operatorname{Hyp}\left(\mathcal{T}_{v}\right)\right.$ and $\exists \overline{C^{o}} \exists \overline{R C}$ with $C^{o} \subseteq \overline{C^{o}} \subseteq \operatorname{Var}\left(\mathcal{T}_{v}\right)$ and $R C \subseteq \overline{R C} \subseteq C l\left(\mathcal{T}_{v}\right)$, s.t.

1. $\bar{S} \cap \overline{C^{o}}=\emptyset, \perp \notin \overline{C^{o}}$, and $\operatorname{Man}\left(\mathcal{T}_{v}\right) \subseteq \bar{S} \cup \overline{C^{o}}$.

2. $\forall r \in \overline{R C}$, head $(r) \in \overline{C^{o}}$ and $\forall p \in \operatorname{body}(r) \cap \operatorname{Var}\left(\mathcal{T}_{v}\right)$ : either $p \in \bar{S}$ or $p \in \overline{C^{o}}$ with $p<\operatorname{head}(r)$. 
3. $R O=\left\{r \in C l(v) \mid \operatorname{body}(r) \cap \operatorname{Var}\left(\mathcal{T}_{v}\right) \nsubseteq \bar{S} \cup \overline{C^{o}}\right\} \cup\{r \in C l(v) \mid \operatorname{head}(r) \in$ $\left.S \cup C^{o}\right\}$ and $\forall r \in C l\left(\mathcal{T}_{v}\right) \backslash C l(v)$, if head $(r) \notin \bar{S} \cup \overline{C^{o}}$ then body $(r) \not \subset \bar{S} \cup \overline{C^{o}}$. 4. $\Delta C=\left\{p \in C^{o} \mid r \in \overline{R C}\right.$, head $\left.(r)=p\right\}$ and $\forall p \in \overline{C^{o}} \backslash C^{o}, \exists r \in \overline{R C}$ with $\operatorname{head}(r)=p$.

Then, occurrences of the ground facts solve $\left(v, S, i, C^{o}, R C, \Delta C, R O, j\right)$ in the lfp of \#HORN-ABDUCTION are determined as follows:

Property C. If $\operatorname{Sol}\left(v, S, C^{o}, R C, \Delta C, R O\right)=\emptyset$ then no atom solve $\left(v, S,{ }_{-}, C^{o}, R C\right.$, $\left.\triangle C, R O,{ }_{-}\right)$is in the lfp of \#Horn-AbduCtion. On the other hand, if $\operatorname{Sol}\left(v, S, C^{o}\right.$, $R C, \Delta C, R O) \neq \emptyset$ then the following conditions are fulfilled:

(a) A fact solve $\left(v, S,{ }_{-}, C^{o}, R C, \Delta C, R O, j\right)$ is in the lfp of \#Horn-ABDUCTION iff $\left|\operatorname{Sol}\left(v, S, C^{o}, R C, \Delta C, R O\right)\right|=j$.

(b) For any further tuple of values $\left(C_{1}^{o}, R C_{1}, \Delta C_{1}, R O_{1}\right)$ we have $\operatorname{Sol}\left(v, S, C^{o}\right.$, $R C, \Delta C, R O)=\operatorname{Sol}\left(v, S, C_{1}^{o}, R C_{1}, \Delta C_{1}, R O_{1}\right)$ iff there exists an index $i$ and a value $j$, s.t. there are facts solve $\left(v, S, i, C^{o}, R C, \Delta C, R O, j\right)$ and solve $\left(v, S, i, C_{1}^{o}, R C_{1}\right.$, $\left.\Delta C_{1}, R O_{1}, j\right)$ in the lfp of \#HORN-ABDUCTION.

The other predicates have the following intended meaning: $\operatorname{svar}(v, S)$ is used to select sets of hypotheses. It is true for every subset $S \subseteq \operatorname{Hyp}(v)$. A fact explains $(v, X)$ is in the lfp iff $\operatorname{Man}(v) \subseteq X$. These two predicates are only used to ease the notation at the leaf nodes of $\mathcal{T}$. The remaining predicates consistent, outside, inside, and derived take care of the conditions $2-4$ of the definition of $\operatorname{Sol}\left(v, S, C^{o}, R C, \Delta C, R O\right)$ in the following way: A fact consistent $\left(R C, S, C^{o}, X\right)$ is in the lfp iff $\forall r \in R C$ we have $\operatorname{head}(r) \in C^{o}$ and $\forall p \in \operatorname{body}(r) \cap X$ it holds that either $p \in S$ or $p \in C^{o}$ with $p<$ head $(r)$, i.e. the rules in $R C$ are only used to derive greater variables from smaller ones (plus variables from $S$ ), cf. condition 2 above. A fact outside $(R O, R, X)$ is in the lfp iff $R O=\{r \in R \mid \operatorname{body}(r) \cap X \neq \emptyset\}$. Hence, for $X \subseteq V \backslash \mathcal{S}^{+}$, the rules in $R O$ do not constitute a contradiction with the closedness of $\mathcal{S}^{+}$because their bodies have a variable of $X$ (and, therefore, outside $\mathcal{S}^{+}$) in their body. A fact inside $(R O, R, X)$ is in the lfp iff $R O=\{r \in R \mid$ head $(r) \in X\}$. Hence, for $X \subseteq \mathcal{S}^{+}$, the rules in $R O$ do not constitute a contradiction with the closedness of $\mathcal{S}^{+}$because their head is inside this set. A fact derived $\left(\Delta C, C^{o}, R C\right)$ means that $\Delta C$ contains those variables of $C^{o}$ for which $R C$ already contains the rule which is used in the last step of the derivation, i.e., $\Delta C=\left\{q \in C^{o} \mid r \in R C, q=h e a d(r)\right\}$. Analogously to Theorems 2 and 3, the \#HORN-ABDUCTION-program in Figure 5 has the following properties:

Theorem 4. Let $\mathcal{P}=\langle V, H, M, \mathcal{C}\rangle$ be an instance of \#HORN-ABDUCTION, encoded by a $\tau_{t d}$-structure $\mathcal{A}_{t d}$. Then, count $(j)$ with $j \geq 1$ is in the lfp of the \#HORN-ABDUCTION-program evaluated on $\mathcal{A}_{t d}$ iff the PAP $\mathcal{P}$ is solvable and has $j$ solutions. Moreover, both the construction of the $\tau_{t d}$-structure $\mathcal{A}_{t d}$ and the evaluation of the program take time $\mathcal{O}(f(t w(\mathcal{P})) *\|\mathcal{P}\|)$ for some function $f$, if we assume constant runtime for the arithmetic operations.

\section{Experimental Evaluation}

A practical evaluation of the monadic datalog approach presented in earlier work [7] is still missing. So far, datalog programs (like the ones established in $[7,8]$ ) only served as a "specification" for an implementation in $\mathrm{C}++$, rather than being used as a method 


\begin{tabular}{|r|r|r|r|r|r|r|r|}
\hline tw & \# vars $\#$ clauses & \# nodes & \# models & Haskell & datalog \\
\hline 3 & 75 & 25 & 220 & $2.1 \mathrm{E} 13$ & 0.00 & 5.67 \\
3 & 150 & 50 & 439 & $2.2 \mathrm{E} 25$ & 0.00 & 22.22 \\
3 & 300 & 100 & 949 & $4.6 \mathrm{E} 54$ & 0.00 & 177.90 \\
\hline 4 & 75 & 25 & 214 & $9.8 \mathrm{E} 11$ & 0.00 & 6.07 \\
4 & 150 & 50 & 453 & $9.0 \mathrm{E} 28$ & 0.00 & 22.72 \\
4 & 300 & 100 & 950 & $2.6 \mathrm{E} 52$ & 0.01 & 233.24 \\
\hline 5 & 300 & 100 & 913 & $2.3 \mathrm{E} 51$ & 0.01 & 166.72 \\
\hline 6 & 300 & 100 & 981 & $1.7 \mathrm{E} 53$ & 0.02 & 141.20 \\
\hline 7 & 300 & 100 & 979 & $3.6 \mathrm{E} 52$ & 0.04 & 259.97 \\
\hline 10 & 309 & 103 & 1044 & $5.1 \mathrm{E} 48$ & 4.12 & 2841.10 \\
\hline
\end{tabular}

Table 1. Processing Time in sec. for \#SAT.

of its own for solving the problem. However, using the datalog approach directly would be very appealing, for instance, for rapid prototyping. Below we report on some first lessons learned when experimenting with implementations of the \#SAT program.

When evaluating the \#SAT-program on a datalog engine, several obstacles have to be overcome: First, encodings for the non-standard datalog operations, especially those for set arithmetic, are non-trivial and must be done very carefully (avoiding the introduction of cycles, etc.). A recent extension of the DLV-system [11], which is called DLV-Complex (see http://www.mat.unical.it/dlv-complex), provides special built-in predicates for set arithmetic. Our experiments showed that such built-ins normally lead to a better performance than a direct realization of the \#SAT-program in "pure" datalog. Another interesting observation was that the DLV-system did not recognize that the solve()-predicate can be evaluated without any cycles by a bottom-up traversal of the tree-decomposition. We therefore relaxed the separation of the program and the data and generated the programs using predicates solve $v()$, for each node $v$ in the tree-decomposition instead of having $v$ as an argument in solve () - thus making the acyclicity explicit. This led to a significant speed-up. Note that we could have put more and more computation tasks into the generation of the datalog program. However, to keep the method generic (w.r.t. different problems) we restricted ourselves to exploit only structural information, i.e. the shape of the tree decomposition. Further, DLV is handicapped in the way that no values bigger than $10^{10}$ can be processed.

We carried out experiments with two implementations of our \#SAT program: one executing the datalog program directly on DLV-complex (compiling the tree structure into the program as discussed above) and one using a general-purpose, Turing complete programming language (in contrast to $[7,8]$, we used Haskell rather than $\mathrm{C}++$, because we found it more convenient). Table 1 shows a glimpse of our results for various values of the treewidth (tw), number of variables (\# vars), clauses (\# clauses) and nodes in the tree decomposition (\# nodes). The experiments were done on a recent Core2Duo processor with $2 \mathrm{~GB}$ of RAM and two cores at $1.86 \mathrm{GHz}$. The time was measured with the Unix tool "time". DLV was called with the default optimization parameters. Haskell was compiled with increased optimization levels. Comparing a compiled program with an interpreted program might be "unfair", but the Haskell program does not need to be recompiled when the tree changes whereas the DLV program has to be generated for each instance. 
In theory, our \#SAT algorithm specified in terms of a datalog program is fixedparameter linear whenever the program is evaluated in an "optimal" way. This is what our Haskell implementation does. For the time being, it is unclear how the design of the datalog program (or the underlying datalog engine) has to be changed such that the datalog engine yields similar results. This is subject of ongoing research. Nevertheless the datalog approach scales reasonably for instances of medium size. Therefore, already now, datalog engines can be employed as tools for rapid prototyping and to verify specifications, which are planned to be realized by a program in another language.

\section{Conclusion}

We have shown that the monadic datalog approach of [7] can be extended to counting problems defined via MSO. It should be noted that - as opposed to $[13,14]$ - our ultimate goal is not an efficient algorithm for the \#S AT problem. Instead we are aiming at a general-purpose method which allows us to systematically turn theoretical tractability results based on Courcelle's Theorem and generalizations thereof into efficient computations. The experiments with our proof-of-concept implementation demonstrate that our goal is realistic even though there is still a lot of work ahead of us.

Analogously to $[13,14]$, our datalog programs ultimately follow a dynamic programming approach. This is not surprising if we keep the crucial observation underlying Courcelle's Theorem in mind: Consider a structure $\mathcal{A}$ with tree decomposition $\mathcal{T}$ and some node $s$ in $\mathcal{T}$. If a domain element $a$ in some bag above $s$ and an element $b$ below $s$ jointly occur in some tuple in $\mathcal{A}$ then - by the definition of tree decompositions $-b$ also occurs in the bag of $s$. Hence, the essential properties of the substructure induced by the subtree rooted at $s$ can be described in terms of the elements in the bag of $s-$ without taking the concrete form of the subtree rooted at $s$ into account. Indeed, our \#S AT program behaves very similar to the dynamic programming algorithm in [14] for the incidence graph. Nevertheless, we find the declarative style of datalog appealing and it has proved convenient in tackling not only \#P problems but also the \#NP-problem \#CIRCUMSCRIPTION. Moreover, the use of datalog allows us to take advantage of all future improvements of datalog engines, which is a very active research area [16].

As future work in this area, we are planning to prove a general expressivity result as to how monadic datalog has to be extended in order to be applicable to any MSO-based counting problem over structures with bounded treewidth. Moreover, we also want to integrate further extensions of Courcelle's Theorem (like sum, minimum, and maximum, which are studied in [3]) into the monadic datalog approach of [7]. As far as our implementation on top of DLV is concerned, we have already identified some directions of future work in Section 6. Note that we have so far used DLV only as a "black box" by converting a \#SAT problem instance plus the extended datalog program for \#SAT into the DLV syntax. Integrating some of the extensions into the datalog system itself (e.g., an efficient implementation of the reduce-predicate in Figures 4 and 5 via hash tables) would clearly help to improve the performance.

\section{References}

1. Courcelle, B.: Graph rewriting: An algebraic and logic approach. In: Handbook of Theoretical Computer Science, Volume B. Elsevier Science Publishers (1990) 193-242 
2. Gottlob, G., Pichler, R., Wei, F.: Bounded treewidth as a key to tractability of knowledge representation and reasoning. In: Proc. AAAI'06. (2006) 250-256

3. Arnborg, S., Lagergren, J., Seese, D.: Easy problems for tree-decomposable graphs. J. Algorithms 12 (1991) 308-340

4. Flum, J., Frick, M., Grohe, M.: Query evaluation via tree-decompositions. J. ACM 49 (2002) 716-752

5. Frick, M., Grohe, M.: The complexity of first-order and monadic second-order logic revisited. In: Proc. LICS'02. (2002) 215-224

6. Grohe, M.: Descriptive and parameterized complexity. In: Proc. CSL'99. Volume 1683 of LNCS. (1999) 14-31

7. Gottlob, G., Pichler, R., Wei, F.: Monadic datalog over finite structures with bounded treewidth. In: Proc. PODS'07. (2007) 165-174

8. Gottlob, G., Pichler, R., Wei, F.: Abduction with bounded treewidth: From theoretical tractability to practically efficient computation. In: Proc. AAAI'08. (2008) 1541-1546

9. Durand, A., Hermann, M., Kolaitis, P.G.: Subtractive reductions and complete problems for counting complexity classes. Theor. Comput. Sci. 340 (2005) 496-513

10. Hermann, M., Pichler, R.: Counting complexity of propositional abduction. In: Proc. IJCAI 2007. (2007) 417-422

11. Leone, N., Pfeifer, G., Faber, W., Eiter, T., Gottlob, G., Perri, S., Scarcello, F.: The DLV system for knowledge representation and reasoning. ACM Trans. Comput. Log. 7 (2006) 499-562

12. Courcelle, B., Makowsky, J.A., Rotics, U.: On the fixed parameter complexity of graph enumeration problems definable in monadic second-order logic. Discrete Applied Mathematics 108 (2001) 23-52

13. Fischer, E., Makowsky, J.A., Ravve, E.V.: Counting truth assignments of formulas of bounded tree-width or clique-width. Discrete Applied Mathematics 156 (2008) 511-529

14. Samer, M., Szeider, S.: Algorithms for propositional model counting. In: Proc. LPAR'07. Volume 4790 of LNCS., Springer (2007) 484-498

15. Bodlaender, H.L.: A linear-time algorithm for finding tree-decompositions of small treewidth. SIAM J. Comput. 25 (1996) 1305-1317

16. Gebser, M., Liu, L., Namasivayam, G., Neumann, A., Schaub, T., Truszczyński, M.: The first answer set programming system competition. In: Proc. LPNMR'07. Volume 4483 of LNCS., Springer (2007) 3-17 


\section{A Correctness Proof of the \#SAT Program}

The only part missing in the proof of Theorem 2 is that the predicate sat () indeed has the intended meaning, i.e. Property A holds. We prove the "if"- and the "only if"-direction separately, and proceed in each part by structural induction on the tree decomposition:

"only if"-direction. Let the fact $\operatorname{sat}\left(v, P, N, C_{u}, j\right)$ be in the lfp of the program. We have to show that $j$ is an integer with $j \geq 1$ and the set $S\left(v, P, N, C_{u}\right)$ indeed has the cardinality $\left|S\left(v, P, N, C_{u}\right)\right|=j$.

base case. Let $v$ be a leaf node. Then $j=1$ and the facts $\operatorname{partition}(X, P, N)$ with $X=\operatorname{Var}(v)$ and $\operatorname{true}\left(P, N, C_{u}, C\right)$ with $C=C l(v)$ are also in the lfp. This means, $P$ and $N$ form a partition of $\operatorname{Var}(v)$ and therefore represent a truth value assignment $(P, N)$. Since $v$ is a leaf node, $\operatorname{Var}(v)=\operatorname{Var}\left(\mathcal{T}_{v}\right)$ and thus the only extension $J$ of $(P, N)$ to $\operatorname{Var}\left(\mathcal{T}_{v}\right)$ is $(P, N)$ itself. Furthermore the atom true ensures that $C_{u}$ contains exactly those clauses $c \in C l(v)$, for which $c$ is true in $J$. Therefore $S\left(v, P, N, C_{u}\right)=$ $\{J\}$ and hence, $\left|S\left(v, P, N, C_{u}\right)\right|=1=j$.

induction step. We distinguish between the five different types of inner nodes of $\mathcal{T}$ assuming that Property A holds for all child nodes.

(1) Let $v$ be a variable removal node with removal of variable $x$ and let $v_{1}$ denote the child of $v$. We have to distinguish three cases. In the first case the two atoms $\operatorname{sat}\left(v_{1}, P \uplus\right.$ $\left.\{x\}, N, C_{u}, j_{1}\right)$ and $\operatorname{sat}\left(v_{1}, P, N \uplus\{x\}, C_{u}, j_{2}\right)$ are both in the lfp and $j=j_{1}+j_{2}$. By the induction hypothesis both $j_{1} \geq 1$ and $j_{2} \geq 1$. Furthermore the sets $S_{1}=$ $S\left(v_{1}, P \uplus\{x\}, N, C_{u}\right)$ and $S_{2}=S\left(v_{1}, P, N \uplus\{x\}, C_{u}\right)$ have cardinality $\left|S_{1}\right|=j_{1}$ and $\left|S_{2}\right|=j_{2}$ respectively. By definition of $S=S\left(v, P, N, C_{u}\right)$ it holds that $S_{1} \subseteq S$ and $S_{2} \subseteq S$. Note that $S_{1} \cap S_{2}=\emptyset$ since their elements differ on the truth value assignment of $x$. Let $J \in S$ be an extension of $(P, N)$ to $\operatorname{Var}\left(\mathcal{T}_{v}\right)$. Then either $J(x)=$ true or $J(x)=$ false. But then by definition of $S$, either $J \in S_{1}$ or $J \in S_{2}$ and therefore $S=S_{1} \uplus S_{2}$. Hence $|S|=j_{1}+j_{2}=j \geq 2$.

In the second case $\operatorname{sat}\left(v_{1}, P \uplus\{x\}, N, C_{u}, j_{1}\right)$ is in the lfp but the fact $\operatorname{sat}\left(v_{1}, P, N \uplus\right.$ $\left.\{x\}, C, j_{2}\right)$ is not and $j=j_{1}$. By the induction hypothesis $j_{1} \geq 1$. Furthermore the sets $S_{1}=S\left(v_{1}, P \uplus\{x\}, N, C_{u}\right)$ and $S_{2}=S\left(v_{1}, P, N \uplus\{x\}, C_{u}\right)$ have cardinality $\left|S_{1}\right|=j_{1}$ and $\left|S_{2}\right|=0$ respectively. By definition of $S=S\left(v, P, N, C_{u}\right)$ it holds that $S_{1} \subseteq S$. Let $J \in S$ be an extension of $(P, N)$ to $\operatorname{Var}\left(\mathcal{T}_{v}\right)$. Then either $J(x)=$ true or $J(x)=$ false. But then by definition of $S$, either $J \in S_{1}$ or $J \in S_{2}$. Since $S_{2}=\emptyset$, $S=S_{1}$ and $|S|=j_{1}=j \geq 1$.

In the third case $\operatorname{sat}\left(v_{1}, P, N \uplus\{x\}, C_{u}, j_{1}\right)$ is in the lfp but the predicate $\operatorname{sat}\left(v_{1}, P \uplus\right.$ $\left.\{x\}, N, C_{u}, j_{2}\right)$ is not and $j=j_{1}$. This case is treated analogously to the second one.

(2) Let $v$ be a clause removal node with removal of clause $c$ and let $v_{1}$ denote the child of $v$. Then the fact $\operatorname{sat}\left(v_{1}, P, N, C_{u} \uplus\{c\}, j\right)$ is also in the lfp. By the induction hypothesis the set $S_{1}=S\left(v_{1}, P, N, C_{u} \uplus\{c\}\right)$ has cardinality $\left|S_{1}\right|=j \geq 1$. Note that $\left(C l\left(\mathcal{T}_{v_{1}}\right) \backslash C l\left(v_{1}\right)\right) \cup C_{u} \uplus\{c\}=\left(C l\left(\mathcal{T}_{v}\right) \backslash C l(v)\right) \cup C_{u}$ and $C l\left(v_{1}\right) \backslash\left(C_{u} \uplus\{c\}\right)=$ $C l(v) \backslash C_{u}$. Hence by the definition of $S=S\left(v, P, N, C_{u}\right)$ it holds that $S_{1}=S$ and therefore $|S|=j \geq 1$.

(3) Let $v$ be a variable introduction node with introduction of variable $x$ and let $v_{1}$ denote the child of $v$. We have to distinguish two cases depending on whether $x \in P$ or $x \in N$. In the first case $P$ is of the form $P^{\prime} \uplus\{x\}$ with a set $P^{\prime} \subseteq \operatorname{Var}\left(v_{1}\right)$ and in the second case $N$ is of the form $N^{\prime} \uplus\{x\}$ with a set $N^{\prime} \subseteq \operatorname{Var}\left(v_{1}\right)$. We only treat the first 
case here. The second one is symmetrical. In the first case the atom $\operatorname{true}\left(\{x\}, \emptyset, C^{\prime}, C\right)$ with $C=C l(v)$ is also in the lfp. This means $C^{\prime}$ contains exactly those clauses in $C l(v)$ where the variable $x$ occurs in unnegated form. Additionally the following mutually distinct facts are in the lfp: $\operatorname{sat}\left(v_{1}, P^{\prime}, N, C_{1}, j_{1}\right), \operatorname{sat}\left(v_{1}, P^{\prime}, N, C_{2}, j_{2}\right), \ldots$, $\operatorname{sat}\left(v_{1}, P^{\prime}, N, C_{n}, j_{n}\right)$ with $n \geq 1$. In particular, it holds that $C_{k} \neq C_{l}$ for all $k \neq l$ with $k, l \in\{1, \ldots, n\}$. Furthermore we have $\sum_{k=1}^{n} j_{k}=j$ and it holds that $C_{k} \cup C^{\prime}=C_{u}$ for $k=1, \ldots, n$. By the induction hypothesis the sets $S_{k}=S\left(v_{1}, P^{\prime}, N, C_{k}\right)$ have cardinality $\left|S_{k}\right|=j_{k} \geq 1$. We define a function $f$ that transforms a truth value assignment $J$ on $\operatorname{Var}\left(\mathcal{T}_{v_{1}}\right)$ into a truth value assignment on $\operatorname{Var}\left(\mathcal{T}_{v}\right)$ in the following way: $f(J)(y)=J(y)$ for all $y \in \operatorname{Var}\left(\mathcal{T}_{v_{1}}\right)$ and $f(J)(x)=$ true. Now let the sets $S_{k}^{\prime}=\left\{f(J) \mid J \in S_{k}\right\}$. Of course it holds that $\left|S_{k}\right|=\left|S_{k}^{\prime}\right|$. By definition of $S=S\left(v, P, N, C_{u}\right)$ it is true that for all $S_{k}^{\prime}$ we have $S_{k}^{\prime} \subseteq S$.

We claim that $S_{k}^{\prime} \cap S_{l}^{\prime}=\emptyset$ for all $k \neq l$. To see this, let $J^{\prime} \in S_{k}^{\prime} \cap S_{l}^{\prime}$ and $k \neq l$. This means, there exists a truth value assignment $J$ on $\operatorname{Var}\left(\mathcal{T}_{v_{1}}\right)$ with $J^{\prime}=f(J)$. Therefore it holds that $J \in S_{k} \cap S_{l}$. But then, by the construction of $S_{k}$ and $S_{l}$, we have that $C_{k}=C_{l}$ which implies that $k=l$ and therefore is a contradiction to the assumption that $k \neq l$.

Now let $J^{\prime} \in S$ be an extension of $(P, N)$ to $\operatorname{Var}\left(\mathcal{T}_{v}\right)$. We claim that there exists a $k$ for which $J^{\prime} \in S_{k}^{\prime}$. To see this, let $J$ be the extension of $\left(P^{\prime}, N\right)$ to $\operatorname{Var}\left(\mathcal{T}_{v_{1}}\right)$ for which $J^{\prime}=f(J)$. Now let $C_{k} \subseteq C l\left(v_{1}\right)$ contain exactly those clauses $c \in C l\left(v_{1}\right)$, for which $c$ is true in $J$. But then it holds that $J \in S_{k}$ and therefore $J^{\prime} \in S_{k}^{\prime}$. Hence $S$ has the form $S=\biguplus_{k=1}^{n} S_{k}^{\prime}$ with cardinality $|S|=\left(\sum_{k=1}^{n} j_{k}\right)=j \geq n$.

(4) Let $v$ be a clause introduction node with introduction of clause $c$ and let $v_{1}$ denote the child of $v$. Then the facts $\operatorname{sat}\left(v_{1}, P, N, C_{1}, j\right)$ and $\operatorname{true}\left(P, N, C_{2},\{c\}\right)$ with $C_{u}=$ $C_{1} \cup C_{2}$ are also in the lfp. The latter one means that $C_{2}=\{c\}$ if $\left.c\right|_{\operatorname{Var}(v)}$ is true in $(P, N)$ and $C_{2}=\emptyset$ otherwise. Note that by the connectedness condition, $\left.c\right|_{\operatorname{Var}\left(\mathcal{T}_{v}\right)}=$ $\left.c\right|_{\operatorname{Var}(v)}$. Hence, the truth value of $\left.c\right|_{\operatorname{Var}\left(\mathcal{T}_{v}\right)}$ in any extension of $(P, N)$ to $\operatorname{Var}\left(\mathcal{T}_{v}\right)$ coincides with the truth value of $\left.c\right|_{\operatorname{Var}(v)}$ in $(P, N)$. By the induction hypothesis the set $S_{1}=S\left(v_{1}, P, N, C_{1}\right)$ has cardinality $\left|S_{1}\right|=j \geq 1$.

We claim that $S_{1} \subseteq S$ with $S=S\left(v, P, N, C_{u}\right)$. To see this, first consider the case that $\left.c\right|_{\operatorname{Var}(v)}$ is false in $(P, N)$. Hence, $C_{2}=\emptyset$ and, therefore, $C_{u}=C_{1}$. But then, by the definition of $S$, it holds that $S=S_{1}$. Now consider the case that $\left.c\right|_{\operatorname{Var}(v)}$ is true in $(P, N)$. Then, $C_{2}=\{c\}$ and $C_{u}$ is of the form $C=C_{1} \cup\{c\}$. Again, by the definition of $S$, we have $S=S_{1}$. Hence $S$ has cardinality $|S|=j \geq 1$.

(5) Let $v$ be a branch node and let $v_{1}$ and $v_{2}$ denote the children of $v$. Then the following mutually distinct pairs of atoms are also in the lfp: $\left\langle\operatorname{sat}\left(v_{1}, P, N, C_{11}, j_{11}\right)\right.$, $\left.\operatorname{sat}\left(v_{2}, P, N, C_{12}, j_{12}\right)\right\rangle, \ldots,\left\langle\operatorname{sat}\left(v_{1}, P, N, C_{n 1}, j_{n 1}\right)\right.$, sat $\left.\left(v_{2}, P, N, C_{n 2}, j_{n 2}\right)\right\rangle$ with $n \geq 1$ and $\sum_{k=1}^{n} j_{k 1} * j_{k 2}=j$ and it holds that $C_{k 1} \cup C_{k 2}=C_{u}$ for $k=1, \ldots, n$. By the induction hypothesis the sets $S_{k 1}=S\left(v_{1}, P, N, C_{k 1}\right)$ and $S_{k 2}=S\left(v_{2}, P, N, C_{k 2}\right)$ have cardinality $\left|S_{k 1}\right|=j_{k 1} \geq 1$ and $\left|S_{k 2}\right|=j_{k 2} \geq 1$ respectively. We define a function $f$ that combines an extension $J_{1}$ of $(P, N)$ to $\operatorname{Var}\left(\mathcal{T}_{v_{1}}\right)$ and an extension $J_{2}$ of $(P, N)$ to $\operatorname{Var}\left(\mathcal{T}_{v_{2}}\right)$ into an extension of $(P, N)$ to $\operatorname{Var}\left(\mathcal{T}_{v}\right)$ in the following way:

$$
f\left(J_{1}, J_{2}\right)(x)= \begin{cases}(P, N)(x) & \text { if } x \in \operatorname{Var}(v) \\ J_{1}(x) & \text { if } x \in \operatorname{Var}\left(\mathcal{T}_{v_{1}}\right) \backslash \operatorname{Var}(v) \\ J_{2}(x) & \text { if } x \in \operatorname{Var}\left(\mathcal{T}_{v_{2}}\right) \backslash \operatorname{Var}(v)\end{cases}
$$


Note that $f\left(J_{1}, J_{2}\right)$ is proper defined for all $x \in \operatorname{Var}\left(\mathcal{T}_{v}\right)$ since by the connectedness condition $\operatorname{Var}\left(\mathcal{T}_{v_{1}}\right) \cap \operatorname{Var}\left(\mathcal{T}_{v_{2}}\right)=\operatorname{Var}(v)$.

Now let $J_{k 1}$ and $J_{k 2}$ be two arbitrary elements of $S_{k 1}$ and $S_{k 2}$ respectively. We claim that then $f\left(J_{k 1}, J_{k 2}\right) \in S$ with $S=S\left(v, P, N, C_{u}\right)$. To see this, note that for each $c \in\left(C l\left(\mathcal{T}_{v}\right) \backslash C l(v)\right) \cup C_{u}$ it holds either that $c \in\left(C l\left(\mathcal{T}_{v_{1}}\right) \backslash C l\left(v_{1}\right)\right) \cup C_{k 1}$ or that $c \in$ $\left(C l\left(\mathcal{T}_{v_{2}}\right) \backslash C l\left(v_{2}\right)\right) \cup C_{k 2}$. Therefore $c$ is true in $f\left(J_{k 1}, J_{k 2}\right)$. On the other hand, for each $c \in C l(v) \backslash C_{u}$, the restriction $\left.c\right|_{\operatorname{Var}\left(\mathcal{T}_{v_{1}}\right)}$ is false in $J_{k 1}$ and the restriction $\left.c\right|_{\operatorname{Var}\left(\mathcal{T}_{v_{2}}\right)}$ is false in $J_{k 2}$. Hence $\left.c\right|_{\operatorname{Var}\left(\mathcal{T}_{v}\right)}$ is false in $f\left(J_{k 1}, J_{k 2}\right)$. But then, by the definition of $S$, it holds that $f\left(J_{k 1}, J_{k 2}\right) \in S$. Since there are $j_{k 1}$ possibilities to choose $J_{k 1}$ and $j_{k 2}$ possibilities to choose $J_{k 2}$, there are $j_{k 1} * j_{k 2}$ different extensions $f\left(J_{k 1}, J_{k 2}\right)$ of $(P, N)$ to $\operatorname{Var}\left(\mathcal{T}_{v}\right)$ for each $k=1, \ldots, n$.

Let $\left\langle S_{k 1}, S_{k 2}\right\rangle$ and $\left\langle S_{l 1}, S_{l 2}\right\rangle$ be two arbitrary pairs with $k, l \in\{1, \ldots, n\}$ and $k \neq l$. It is easy to see, that the extensions $f\left(J_{k 1}, J_{k 2}\right)$ and $f\left(J_{l 1}, J_{l 2}\right)$ of $(P, N)$ to $\operatorname{Var}\left(\mathcal{T}_{v}\right)$ that can be constructed by combining arbitrary $J_{k 1} \in S_{k 1}, J_{k 2} \in S_{k 2}$ and $J_{l 1} \in S_{l 1}, J_{l 2} \in S_{l 2}$ are indeed mutually distinct. Otherwise it holds that $J_{k 1}=J_{l 1}$ and $J_{k 2}=J_{l 2}$. But by the definition of $S_{k 1}, S_{k 2}, S_{l 1}$ and $S_{l 2}$ this means that $S_{k 1}=S_{l 1}$ and $S_{k 2}=S_{l 2}$. This implies that the corresponding pairs of facts $\left\langle\operatorname{sat}\left(v_{1}, P, N, C_{k 1}, j_{k 1}\right)\right.$, $\left.\operatorname{sat}\left(v_{2}, P, N, C_{k 2}, j_{k 2}\right)\right\rangle$ and $\left\langle\operatorname{sat}\left(v_{1}, P, N, C_{l 1}, j_{l 1}\right)\right.$, sat $\left.\left(v_{2}, P, N, C_{l 2}, j_{l 2}\right)\right\rangle$ are equal, which in turn implies that $k=l$. Therefore we have a contradiction with our assumption that $k \neq l$.

Finally let $J \in S$ be an arbitrary extension of $(P, N)$ to $\operatorname{Var}\left(\mathcal{T}_{v}\right)$. Then there exists an extension $J_{1}$ of $(P, N)$ to $\operatorname{Var}\left(\mathcal{T}_{v_{1}}\right)$ and an extension $J_{2}$ of $(P, N)$ to $\operatorname{Var}\left(\mathcal{T}_{v_{2}}\right)$, s.t. $J=f\left(J_{1}, J_{2}\right)$. Furthermore let $C_{1} \subseteq C_{u}$ contain exactly those clauses $c \in C_{u}$ which are true in $J_{1}$ and let $C_{2} \subseteq C_{u}$ contain exactly those clauses $c \in C_{u}$ which are true in $J_{2}$. Clearly we have $C_{1} \cup C_{2}=C_{u}$. But then the lfp contains a pair of facts $\left\langle\operatorname{sat}\left(v_{1}, P, N, C_{1}, j_{1}\right)\right.$, sat $\left.\left(v_{2}, P, N, C_{2}, j_{2}\right)\right\rangle$ and the truth value assignments $J_{1}$ and $J_{2}$ are contained in the corresponding sets $S\left(v_{1}, P, N, C_{1}\right)$ and $S\left(v_{2}, P, N, C_{2}\right)$ respectively. Therefore and with the arguments above, we have that $S$ has cardinality $|S|=\sum_{k=1}^{n} j_{k 1} * j_{k 2}=j \geq n$.

"if"-direction. Let the cardinality of the set $S=S\left(v, P, N, C_{u}\right)$ be $|S|=j$ with $j \geq 0$. We have to show that the fact $\operatorname{sat}\left(v, P, N, C_{u}, j\right)$ is in the lfp of the program if $j \neq 0$ and that otherwise no fact of the form $\operatorname{sat}\left(v, P, N, C_{u},{ }_{-}\right)$is in the lfp.

base case. Let $v$ be a leaf node and let $j \geq 1$. Since $P$ and $N$ form a partition of $\operatorname{Var}(v)$, the only extension $J \in S$ of $(P, N)$ to $\operatorname{Var}\left(\mathcal{T}_{v}\right)=\operatorname{Var}(v)$ is $(P, N)$ itself. Therefore $j=1$ and the fact partition $(X, P, N)$ with $X=\operatorname{Var}(v)$ is in the lfp of the program. By the definition of $S, C_{u}$ contains exactly those clauses $c \in C l(v)$, for which $\left.c\right|_{\operatorname{Var}(v)}$ is true in $J=(P, N)$. Hence the atom $\operatorname{true}\left(P, N, C_{u}, C\right)$ with $C=\operatorname{cl}(v)$ is in the lfp, which implies that also the fact $\operatorname{sat}\left(v, P, N, C_{u}, 1\right)$ is in the lfp.

On the other hand let $j=0$. This means, $C_{u}$ does not contain exactly those clauses $c \in C l(v)$, for which $\left.c\right|_{\operatorname{Var}(v)}$ is true in $(P, N)$. Therefore the atom $\operatorname{true}\left(P, N, C_{u}, C\right)$ with $C=\operatorname{cl}(v)$ is not in the lfp. Hence no fact of the form $\operatorname{sat}\left(v, P, N, C_{u},{ }_{-}\right)$can be derived.

induction step. We distinguish again between the five different types of inner nodes of $\mathcal{T}$ assuming that Property A holds for all child nodes.

(1) Let $v$ be a variable removal node with removal of variable $x$ and let $v_{1}$ denote the child of $v$. We define two subsets $S_{1}, S_{2} \subseteq S$ in the following way: $S_{1}=\{J \in S \mid$ $J(x)=$ true $\}$ and $S_{2}=\{J \in S \mid J(x)=$ false $\}$ with cardinality $\left|S_{1}\right|=j_{1}$ and $\left|S_{2}\right|=$ 
$j_{2}$. Clearly $S_{1} \uplus S_{2}=S$ and therefore $j=j_{1}+j_{2}$. Note that by definition it holds that $S_{1}=S\left(v_{1}, P \uplus\{x\}, N, C_{u}\right)$ and $S_{2}=S\left(v_{1}, P, N \uplus\{x\}, C_{u}\right)$. Thus by the induction hypothesis, the facts $\operatorname{sat}\left(v_{1}, P \uplus\{x\}, N, C_{u}, j_{1}\right)$ and $\operatorname{sat}\left(v_{1}, P, N \uplus\{x\}, C_{u}, j_{2}\right)$ are in the lfp of the program if $j_{1} \geq 1$ and $j_{2} \geq 1$ respectively. Otherwise no atoms of the form $\operatorname{sat}\left(v_{1}, P \uplus\{x\}, N, C_{u},{ }_{-}\right)$and $\operatorname{sat}\left(v_{1}, P, N \uplus\{x\}, C_{u},{ }_{-}\right)$are in the lfp. Now let $j \geq 1$. We have to distinguish three cases depending on whether none or exactly one of the two cardinalities $j_{1}$ and $j_{2}$ equals 0 . It is easy to see, that in all those cases the corresponding rule implies that $\operatorname{sat}\left(v, P, N, C_{u}, j\right)$ is in the lfp of the program. On the other hand let $j=0$. Since no atoms of the form $\operatorname{sat}\left(v_{1}, P \uplus\{x\}, N, C_{u},{ }_{-}\right)$and $\operatorname{sat}\left(v_{1}, P, N \uplus\{x\}, C_{u},{ }_{-}\right)$are in the lfp, no fact of the form $\operatorname{sat}\left(v, P, N, C_{u},-\right)$ can be derived.

(2) Let $v$ be a clause removal node with removal of clause $c$ and let $v_{1}$ denote the child of $v$. Note that $\left(C l\left(\mathcal{T}_{v}\right) \backslash C l(v)\right) \cup C_{u}=\left(C l\left(\mathcal{T}_{v_{1}}\right) \backslash C l\left(v_{1}\right)\right) \cup C_{u} \uplus\{c\}$ and that $C l(v) \backslash C_{u}=C l\left(v_{1}\right) \backslash\left(C_{u} \uplus\{c\}\right)$. Therefore by definition $S=S_{1}=S\left(v_{1}, P, N, C_{u} \uplus\right.$ $\{c\})$ with $|S|=\left|S_{1}\right|=j$. Assume that $j \geq 1$. Then by the induction hypothesis, $\operatorname{sat}\left(v_{1}, P, N, C_{u} \uplus\{c\}, j\right)$ is in the lfp of the program and therefore $\operatorname{sat}\left(v, P, N, C_{u}, j\right)$ is also in the lfp. On the other hand, let $j=0$. Then by the induction hypothesis, no fact of the form $\operatorname{sat}\left(v_{1}, P, N, C_{u} \uplus\{c\},,_{-}\right)$is in the lfp and therefore no atom of the form sat $\left(v, P, N, C_{u},{ }_{-}\right)$can be derived.

(3) Let $v$ be a variable introduction node with introduction of variable $x$ and let $v_{1}$ denote the child of $v$. We have to distinguish two cases depending on whether $x \in P$ or $x \in N$. We only treat the first case here. The second one is symmetrical. We define a clause set $C(J)$ for all assignments $J \in S$ as follows: $C(J)=\left\{c \in C_{u}\right.$ | $\left.c\right|_{\operatorname{Var}\left(\mathcal{T}_{v_{1}}\right)}$ is true in $\left.\left.J\right|_{\operatorname{Var}\left(\mathcal{T}_{v_{1}}\right)}\right\}$. Thereby $\left.J\right|_{\operatorname{Var}\left(\mathcal{T}_{v_{1}}\right)}$ denotes the restriction of $J$ to $\operatorname{Var}\left(\mathcal{T}_{v_{1}}\right)$. Assuming that $j \geq 1$, we construct a partition $\left\{S_{1}, \ldots, S_{n}\right\}$ of $S$ with $n \geq 1$, s.t. for all $J_{1}, J_{2} \in S_{k}$ with $k=1, \ldots, n$ it holds that $C\left(J_{1}\right)=C\left(J_{2}\right)$ and that for all $J_{1} \in S_{k}$ and $J_{2} \in S_{l}$ with $k \neq l$ it holds that $C\left(J_{1}\right) \neq C\left(J_{2}\right)$. This means we can associate with each partition block $S_{k}$ a clause set $C_{k}=C(J)$ with $J \in S_{k}$. Let the sets $S_{k}$ have cardinality $\left|S_{k}\right|=j_{k} \geq 1$. Clearly we have $j=\sum_{k=1}^{n} j_{k}$. Let $P$ be of the form $P=P^{\prime} \uplus\{x\}$. Then it is easy to see, that $S_{k}=S\left(v_{1}, P^{\prime}, N, C_{k}\right)$. Therefore by the induction hypothesis, the fact $\operatorname{sat}\left(v_{1}, P^{\prime}, N, C_{k}, j_{k}\right)$ is in the lfp of the program. Next let $C^{\prime}$ be defined as $C^{\prime}=\{c \in C l(v): x$ occurs unnegated in $c\}$. Then $C^{\prime} \subseteq C_{u}$ since otherwise there would exist a clause $c \in C l(v) \backslash C_{u}$ with $\left.c\right|_{\operatorname{Var}\left(\mathcal{T}_{v}\right)}$ being true in each $J \in S$. Furthermore $C_{u}=C_{k} \cup C^{\prime}$ holds for each $k=1, \ldots, n$. This is due to the fact that all clauses in $C_{u}$ are true in each $J \in S$. Hence, they are already true in $\left.J\right|_{\operatorname{Var}\left(\mathcal{T}_{v_{1}}\right)}$ or they become true in $J$ because of an unnegated occurrence of $x$. Thus the facts true $\left(\{x\}, \emptyset, C^{\prime}, C\right)$ with $C=C l(v)$ and $C_{k} \cup C^{\prime}=C_{u}$ are in the lfp. This implies that $\operatorname{sat}\left(v, P, N, C_{u}, j\right)$ is also in the lfp.

Now assume that $S=\emptyset$ and let $C^{\prime}$ be defined as above. We claim that for each clause set $C_{k}$ with $C_{k} \cup C^{\prime}=C_{u}$ we have that $S_{k}=S\left(v_{1}, P^{\prime}, N, C_{k}\right)=\emptyset$. To see this, we define an extension $J^{\prime}$ of $(P, N)$ to $\operatorname{Var}\left(\mathcal{T}_{v}\right)$ by extending $J \in S_{k}$ by $J^{\prime}(x)=$ true. But then we have $J^{\prime} \in S$ which is a contradiction to our assumption. Therefore no fact of the form $\operatorname{sat}\left(v_{1}, P^{\prime}, N, C_{k},{ }_{-}\right)$is in the lfp and hence also no atom of the form $\operatorname{sat}\left(v, P, N, C_{u},{ }_{-}\right)$is in the lfp of the program.

(4) Let $v$ be a clause introduction node with introduction of clause $c$ and let $v_{1}$ denote the child of $v$. Let $C_{1}$ be defined as $C_{1}=C_{u} \backslash\{c\}$. Then we have $\left(C l\left(\mathcal{T}_{v_{1}}\right) \backslash C l\left(v_{1}\right)\right) \cup$ $C_{1}=\left(C l\left(\mathcal{T}_{v}\right) \backslash C l(v)\right) \cup C_{1} \subseteq\left(C l\left(\mathcal{T}_{v}\right) \backslash C l(v)\right) \cup C_{u}$ and furthermore $C l\left(v_{1}\right) \backslash$ 
$C_{1}=C l(v) \backslash\left(C_{1} \uplus\{c\}\right) \subseteq C l(v) \backslash C_{u}$. Thus for every $J \in S$ it holds that $J \in S_{1}$ with $S_{1}=\left(v_{1}, P, N, C_{1}\right)$ and hence $S \subseteq S_{1}$. Let $C_{2}$ be defined as $C_{2}=\{c\}$ if $\left.c\right|_{\operatorname{Var}(v)}$ is true in $(P, N)$ and $C_{2}=\emptyset$ otherwise. Now assume that $C_{u}=C_{1} \cup C_{2}$, i.e. $c \in C_{u}$ iff $\left.c\right|_{\operatorname{Var}(v)}$ is true in $(P, N)$. But then clearly for all $J \in S_{1}$ it holds that $J \in S$. Therefore and since $S \subseteq S_{1}$, we have $S=S_{1}$ and $|S|=\left|S_{1}\right|=j$. By the induction hypothesis, if $j \geq 1$ the fact $\operatorname{sat}\left(v_{1}, P, N, C_{1}, j\right)$ is in the lfp of the program. Additionally by our construction, the atoms $\operatorname{true}\left(P, N, C_{2},\{c\}\right)$ and $C_{1} \cup C_{2}=C_{u}$ are also in the lfp. Therefore the fact $\operatorname{sat}\left(v, P, N, C_{u}, j\right)$ will be derived. On the other hand, if $j=0$ no fact of the form $\operatorname{sat}\left(v_{1}, P, N, C_{1},_{-}\right)$is in the lfp. Hence no fact of the form $\operatorname{sat}\left(v, P, N, C_{u},{ }_{-}\right)$can be derived.

The only case left, is the one where $C_{u} \neq C_{1} \cup C_{2}$, i.e. $c \in C_{u}$ iff $\left.c\right|_{\operatorname{Var}(v)}$ is false in $(P, N)$. In this case by definition $S=\emptyset$, i.e. $|S|=0$. But then the fact $C_{1} \cup C_{2}=C_{u}$ is not in the lfp of the program and therefore no atom of the form $\operatorname{sat}\left(v, P, N, C_{u},{ }_{-}\right)$ can be derived.

(5) Let $v$ be a branch node and let $v_{1}$ and $v_{2}$ denote the children of $v$. We define the set Let $\mathcal{C}=\left\{\left(C_{1}^{\prime}, C_{1}^{\prime \prime}\right),\left(C_{2}^{\prime}, C_{2}^{\prime \prime}\right), \ldots,\left(C_{n}^{\prime}, C_{n}^{\prime \prime}\right)\right\}$ of pairs of clause sets as follows: $\mathcal{C}=\left\{\left(C^{\prime}, C^{\prime \prime}\right) \mid C^{\prime} \cup C^{\prime \prime}=C_{u}, S\left(v_{1}, P, N, C^{\prime}\right) \neq \emptyset, S\left(v_{2}, P, N, C^{\prime \prime}\right) \neq \emptyset\right\}$. For every $\alpha \in\{1, \ldots, n\}$, let $S_{\alpha}^{\prime}=S\left(v_{1}, P, N, C_{\alpha}^{\prime}\right)$ and $S_{\alpha}^{\prime \prime}=S\left(v_{2}, P, N, C_{\alpha}^{\prime \prime}\right)$. Moreover, let $j_{\alpha}^{\prime}=\left|S_{\alpha}^{\prime}\right|$ and $j_{\alpha}^{\prime \prime}=\left|S_{\alpha}^{\prime \prime}\right|$. By the induction hypothesis, for every $\alpha \in\{1, \ldots, n\}$, there are pairs of facts in the lfp of the program, which have the form $\left(\operatorname{sat}\left(v_{1}, P, N, C_{\alpha}^{\prime}, j_{\alpha}^{\prime}\right)\right.$, sat $\left.\left(v_{1}, P, N, C_{\alpha}^{\prime \prime}, j_{\alpha}^{\prime \prime}\right)\right)$. Moreover, the lfp does not contain any other facts of this type, i.e., suppose that there exist clause sets $C^{\prime}, C^{\prime \prime}$ with $C^{\prime} \cup C^{\prime \prime}=C_{u}$ and positive integers $j^{\prime}, j^{\prime \prime}$, s.t. the lfp of the program contains two atoms $\operatorname{sat}\left(v_{1}, P, N, C^{\prime}, j^{\prime}\right)$ and $\operatorname{sat}\left(v_{1}, P, N, C^{\prime \prime}, j^{\prime \prime}\right)$, then there exists an index $\alpha \in$ $\{1, \ldots, n\}$ with $\left(C^{\prime}, C^{\prime \prime}\right)=\left(C_{\alpha}^{\prime}, C_{\alpha}^{\prime \prime}\right)$ and $\left(j^{\prime}, j^{\prime \prime}\right)=\left(j_{\alpha}^{\prime}, j_{\alpha}^{\prime \prime}\right)$. Thus, the rule for branch nodes in the \#SAT program produces the fact $\operatorname{sat}\left(v, P, N, C_{u}, k\right)$ with $k=$ $\sum_{\alpha=1}^{n} j_{\alpha}^{\prime} * j_{\alpha}^{\prime \prime}$.

It remains to show that $j=k$, i.e., $k$ indeed denotes the cardinality of the set $S$. Let $S^{\prime}$ be defined as

$$
S^{\prime}=S_{1}^{\prime} \times S_{1}^{\prime \prime} \cup S_{2}^{\prime} \times S_{2}^{\prime \prime} \cup \cdots \cup S_{n}^{\prime} \times S_{n}^{\prime \prime} .
$$

Clearly, any two sets $\left(S_{\alpha}^{\prime} \times S_{\alpha}^{\prime \prime}\right)$ and $\left(S_{\beta}^{\prime} \times S_{\beta}^{\prime \prime}\right)$ with $\alpha \neq \beta$ are disjoint. This follows immediately from the fact that any pair of interpretations $\left(J_{\alpha}^{\prime} \times J_{\alpha}^{\prime \prime}\right) \in\left(S_{\alpha}^{\prime} \times S_{\alpha}^{\prime \prime}\right)$ makes the clauses in $\left(C_{\alpha}^{\prime} \times C_{\alpha}^{\prime \prime}\right)$ true while any pair of interpretations $\left(J_{\beta}^{\prime} \times J_{\beta}^{\prime \prime}\right) \in\left(S_{\beta}^{\prime} \times S_{\beta}^{\prime \prime}\right)$ makes the clauses in $\left(C_{\beta}^{\prime} \times C_{\beta}^{\prime \prime}\right)$ true with $\left(C_{\alpha}^{\prime} \times C_{\alpha}^{\prime \prime}\right) \neq\left(C_{\beta}^{\prime} \times C_{\beta}^{\prime \prime}\right)$. Thus, $\left|S^{\prime}\right|=k$ holds and it suffices to show that there exists a bijection $S^{\prime} \cong S$.

Let $\left(J^{\prime}, J^{\prime \prime}\right)$ be an arbitrary pair of assignments in $S^{\prime}$. Then we write $J^{\prime} \cup J^{\prime \prime}$ to denote the assignment $J$ on $\operatorname{Var}\left(\mathcal{T}_{v}\right)$, s.t. $J$ restricted to $\operatorname{Var}\left(\mathcal{T}_{v_{1}}\right)$ is $J^{\prime}$ and $J$ restricted to $\operatorname{Var}\left(\mathcal{T}_{v_{2}}\right)$ is $J^{\prime \prime}$. By the connectedness condition on tree decompositions and by the fact that $J^{\prime}$ and $J^{\prime \prime}$ coincide on the variables in $\operatorname{Var}(v)=\operatorname{Var}\left(v_{1}\right)=\operatorname{Var}\left(v_{2}\right)$, this assignment $J$ is well-defined. We claim that the desired bijective function $f: S^{\prime} \rightarrow S$ is obtained by defining $f\left(J^{\prime}, J^{\prime \prime}\right)=J^{\prime} \cup J^{\prime \prime}$. Clearly, $f$ is well-defined and $f\left(J^{\prime}, J^{\prime \prime}\right) \in S$ holds.

Moreover, $f$ is surjective and injective for the following reasons: Let $J$ be an arbitrary assignment in $S$ and let $\left.J\right|_{\operatorname{Var}\left(\mathcal{T}_{v_{1}}\right)}\left(\right.$ resp. $\left.J\right|_{\operatorname{Var}\left(\mathcal{T}_{v_{2}}\right)}$ ) denote the restriction of $J$ to the variables in $\operatorname{Var}\left(\mathcal{T}_{v_{1}}\right)$ (resp. $\operatorname{Var}\left(\mathcal{T}_{v_{2}}\right)$ ). Then it clearly holds that we have $\left(\left.J\right|_{\operatorname{Var}\left(\mathcal{T}_{v_{1}}\right)},\left.J\right|_{\operatorname{Var}\left(\mathcal{T}_{v_{2}}\right)}\right) \in S^{\prime}$ and $J=f\left(\left.J\right|_{\operatorname{Var}\left(\mathcal{T}_{v_{1}}\right)},\left.J\right|_{\operatorname{Var}\left(\mathcal{T}_{v_{2}}\right)}\right)$ is true. Hence, $f$ is indeed surjective. Now let $\left(J_{1}^{\prime}, J_{1}^{\prime \prime}\right)$ and $\left(J_{2}^{\prime}, J_{2}^{\prime \prime}\right)$ be two distinct pairs in $S^{\prime}$. It remains 
to show that then $J_{1}^{\prime} \cup J_{1}^{\prime \prime}$ and $J_{2}^{\prime} \cup J_{2}^{\prime \prime}$ are also distinct. Since $\left(J_{1}^{\prime}, J_{1}^{\prime \prime}\right) \neq\left(J_{2}^{\prime}, J_{2}^{\prime \prime}\right)$, we either have $J_{1}^{\prime} \neq J_{2}^{\prime}$ or $J_{1}^{\prime \prime} \neq J_{2}^{\prime \prime}$. W.l.o.g., suppose that $J_{1}^{\prime} \neq J_{2}^{\prime}$. Hence, there exists a variable $x$ in $\operatorname{Var}\left(\mathcal{T}_{v_{1}}\right) \backslash \operatorname{Var}\left(v_{1}\right)$ which has different truth values in $J_{1}^{\prime}$ and $J_{2}^{\prime}$. But then $x$ also has different truth values in $J_{1}^{\prime} \cup J_{1}^{\prime \prime}$ and $J_{2}^{\prime} \cup J_{2}^{\prime \prime}$. Hence, $J_{1}^{\prime} \cup J_{1}^{\prime \prime} \neq J_{2}^{\prime} \cup J_{2}^{\prime \prime}$ indeed holds and, therefore, $f$ is injective.

\section{B Auxiliary Predicates for the \#CIRCuMSCRIPTIOn Program}

A datalog implementation of the predicate reduce for the \#CIRCUMSCRIPTION program plus several auxiliary predicates is given in Figure 6 . The idea of the predicate reduce $\left(v, P, N, i, i_{1}, i_{2}, j\right)$ is to map pairs of indices $\left(i_{1}, i_{2}\right)$ to single indices $i$ and, in particular, to map "equal" (i.e., indistinguishable) pairs of indices $\left(i_{1}, i_{2}\right)$ to the same index $i$. For given values of $v, P, N$, two pairs $\left(i_{1}, i_{2}\right)$ and $\left(i_{1}^{\prime}, i_{2}^{\prime}\right)$ are "equal" iff two facts of the form auxsat $\left(v, i_{1}, i_{2}, P, N, C, j\right)$ and the form auxsat $\left(v, i_{1}^{\prime}, i_{2}^{\prime}, P, N, C, j^{\prime}\right)$ are in the lfp of the program and it holds that auxunsat $\left(v, i_{1}, i_{2}, P, N, P^{\prime}, N^{\prime}, C^{\prime}\right)$ is in the lfp iff the lfp contains an atom auxunsat $\left(v, i_{1}^{\prime}, i_{2}^{\prime}, P, N, P^{\prime}, N^{\prime}, C^{\prime}\right)$.

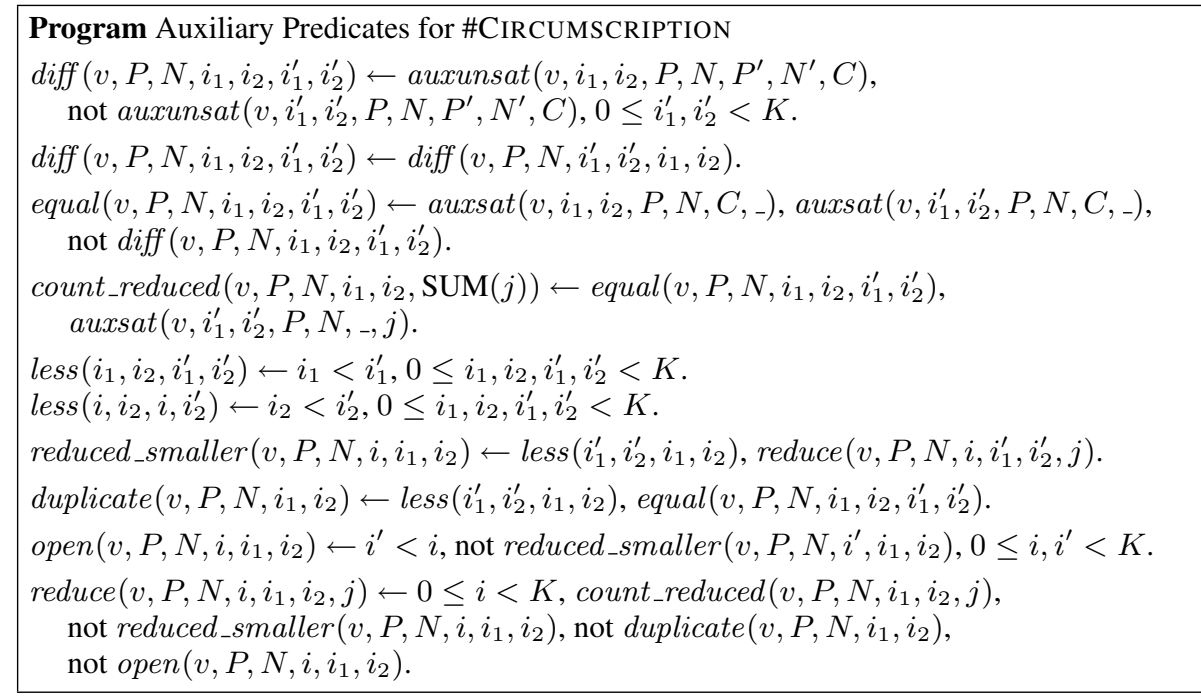

Fig. 6. reduce plus auxiliary predicates for \#CIRCUMSCRIPTION.

In the body of the rule defining reduce $\left(v, P, N, i, i_{1}, i_{2}, j\right)$, we have the condition $0 \leq i<K$, where $K$ denotes the maximum number of non-equal pairs $\left(i_{1}, i_{2}\right)$. This $K$ is a constant which depends solely on the treewidth $w$. A suitable value of $K$ can be obtained by the following considerations: For given values of $v, P, N$, let the bag at $v$ consist of the variables $\operatorname{Var}(v)$ and clauses $C l(v)$ with $\operatorname{Var}(v) \cap C l(v)=\emptyset$ and $|\operatorname{Var}(v) \cup C l(v)| \leq w+1$. For any fact $\operatorname{auxsat}\left(v, i_{1}, i_{2}, P, N, C, j\right)$ we have $C \subseteq$ $C l(v)$. Hence the values of $C$ can be considered as bit vectors of length at most $w+$ 
1. Therefore there are at most $2^{w+1}$ different values for $C$. Furthermore for any fact auxunsat $\left(v, i_{1}, i_{2}, P, N, P^{\prime}, N^{\prime}, C^{\prime}\right)$ we have $\left|P^{\prime}\right|+\left|N^{\prime}\right|=|\operatorname{Var}(v)|$ and $C^{\prime} \subseteq C l(v)$. Hence the values of $\left(P^{\prime}, N^{\prime}, C^{\prime}\right)$ can also be considered as bit vectors of length at most $w+1$. This means for given $v, P, N, C$, there are at most $2^{2^{w+1}}$ different subsets of facts of the form auxunsat $\left(v, i_{1}, i_{2}, P, N, P^{\prime}, N^{\prime}, C^{\prime}\right)$. Putting it all together, we get at most $K=2^{w+1} * 2^{2^{w+1}}$ non-equal pairs $\left(i_{1}, i_{2}\right)$. Thus the index $i$ in the predicate reduce $\left(v, P, N, i, i_{1}, i_{2}, j\right)$ is in $\{0, \ldots, K-1\}$.

The purpose of the predicate $\operatorname{diff}\left(v, P, N, i_{1}, i_{2}, i_{1}^{\prime}, i_{2}^{\prime}\right)$ is to check whether for given values of $v, P, N$, the pairs $\left(i_{1}, i_{2}\right)$ and $\left(i_{1}^{\prime}, i_{2}^{\prime}\right)$ differ in their corresponding auxunsat facts. Recall that it was one of the two requirements for equal pairs, that they do not differ in these facts. Therefore we can use diff to define the predicate equal, where we additionally assure the first requirement for equal pairs: they have to satisfy the same clause set $C$, i.e. there have to be two facts of the form auxsat $\left(v, i_{1}, i_{2}, P, N, C, j\right)$ and $\operatorname{auxsat}\left(v, i_{1}^{\prime}, i_{2}^{\prime}, P, N, C, j^{\prime}\right)$ in the lfp of the program.

Since we are going to group all equal index pairs, we have to update our counter $j$. This is done by the predicate count_reduced, which computes the sum over the counter of the corresponding auxsat facts of all equal index pairs. The intended meaning of the atom less $\left(i_{1}, i_{2}, i_{1}^{\prime}, i_{2}^{\prime}\right)$ is that the pair $\left(i_{1}, i_{2}\right)$ is lexicographically smaller than the pair $\left(i_{1}^{\prime}, i_{2}^{\prime}\right)$. The fact reduced_smaller $\left(v, P, N, i, i_{1}, i_{2}\right)$ denotes that there exists a pair of indices which is smaller than $\left(i_{1}, i_{2}\right)$ and which is already mapped onto the index $i$, i.e. we have to prevent a mapping of $\left(i_{1}, i_{2}\right)$ onto $i$.

The intended meaning of the atom duplicate $\left(v, P, N, i_{1}, i_{2}\right)$ is to indicate that there exists a smaller pair of indices which is equal to $\left(i_{1}, i_{2}\right)$. By allowing the derivation of a reduce fact only for the smallest of all equal pairs of indices, we can assure that there exists only one such fact for each equivalence class. Finally the intended purpose of the fact open $\left(v, P, N, i, i_{1}, i_{2}\right)$ is to assure that we choose the smallest free index $i$ for our mapping, i.e. there exists no smaller index $i^{\prime}$ which we have not yet used in such a mapping.

\section{Correctness Proof of the \#Circumscription Program}

We are going to proof that the predicates sat and unsat indeed have the intended meaning, i.e. Property B holds, by structural induction on the tree decomposition:

base case. Let $v$ be a leaf node. Since $(P, N)$ is a partition of $\operatorname{Var}(v)=\operatorname{Var}\left(\mathcal{T}_{v}\right)$, the only truth value assignment extending $(P, N)$ to $\operatorname{Var}\left(\mathcal{T}_{v}\right)$ is $(P, N)$ itself. Therefore for each set $S=S\left(v, P, N, C_{u}\right)$, the cardinality of $S$ is either $|S|=0$ or $|S|=1$. This implies that if $S \neq \emptyset$, then there is only one possible partition $\left\{S_{0}\right\}$ of $S$ with $S_{0}=S$. Clearly $|S|=1$ iff the predicate $\operatorname{true}\left(P, N, C_{u}, C\right)$ with $C=C l(v)$ is in the lfp iff $\operatorname{sat}\left(v, 0, P, N, C_{u}, 1\right)$ is contained in the lfp.

The implication that $\operatorname{unsat}\left(v, i, P, N,_{-},,_{-},,_{-}\right)$is in the lfp only if also an atom $\operatorname{sat}\left(v, i, P, N,_{-},{ }_{-}\right)$is contained in the lfp is trivially fulfilled, since the latter fact is part of the body of the rule for deriving the first one.

Furthermore note that a fact unsat $\left(v, i, P, N, P^{\prime}, N^{\prime}, C_{u}^{\prime}\right)$ is contained in the lfp $\Leftrightarrow$ the three facts $\operatorname{sat}\left(v, i, P, N, C_{u},{ }_{-}\right)$, sat $\left(v,{ }_{-}, P^{\prime}, N^{\prime}, C_{u}^{\prime}{ }_{-}\right)$and $P^{\prime} \subset P$ are in the lfp of the program $\Leftrightarrow$ there exists a $J \in S\left(v, P, N, C_{u}\right)$ and a $J^{\prime} \in S\left(v, P^{\prime}, N^{\prime}, C_{u}^{\prime}\right)$ with $P^{\prime} \subset P$. 
induction step. We distinguish between the five different types of inner nodes of $\mathcal{T}$ assuming that Property B holds for all child nodes.

Let $v$ be a variable removal node with removal of variable $x$ and let $v_{1}$ denote the child of $v$. First we show that there exists an atom $\operatorname{sat}\left(v,_{-}, P, N, C_{u},_{-}\right)$in the lfp of the program iff $S\left(v, P, N, C_{u}\right) \neq \emptyset$. Assuming that such a fact is in the lfp, a fact of the form auxsat $\left(v,,_{-}, P, N, C_{u},{ }_{-}\right)$is also in the lfp. Therefore at least one of the facts $\operatorname{sat}\left(v_{1},_{-}, P \uplus\{x\}, N, C_{u},{ }_{-}\right)$or $\operatorname{sat}\left(v_{1},_{-}, P, N \uplus\{x\}, C_{u},{ }_{-}\right)$is in the lfp. We only treat the first case here, since the second one is symmetrical. By the induction hypothesis, $S\left(v_{1}, P \uplus\{x\}, N, C_{u}\right) \neq \emptyset$. Note that by the construction of $S$ it holds that $S\left(v_{1}, P \uplus\{x\}, N, C_{u}\right) \subseteq S\left(v, P, N, C_{u}\right)$ and therefore $S\left(v, P, N, C_{u}\right) \neq \emptyset$. Now assume that $S\left(v, P, N, C_{u}\right) \neq \emptyset$. By the construction of $S$, at least one of the following two statements hold: $S\left(v_{1}, P \uplus\{x\}, N, C_{u}\right) \neq \emptyset$ or $S\left(v_{1}, P, N \uplus\{x\}, C_{u}\right) \neq \emptyset$. Again the two cases are symmetrical and we therfore only treat the first one. By the induction hypothesis, a fact $\operatorname{sat}\left(v_{1},{ }_{-}, P \uplus\{x\}, N, C_{u},{ }_{-}\right)$is in the lfp of the program. Therefore also a fact $\operatorname{auxsat}\left(v,_{-},-, P, N, C_{u},-\right)$ and furthermore $\operatorname{sat}\left(v,_{-}, P, N, C_{u},_{-}\right)$is in the lfp.

Next we show that if there exists an atom unsat $\left(v, i, P, N,_{-},,_{-},,_{-}\right)$in the lfp of the program, then there is also an atom $\operatorname{sat}\left(v, i, P, N,,_{-}\right)$in the lfp. Assuming that such an unsat fact exists in the lfp, a predicate $\operatorname{reduce}\left(v, P, N, i, i_{1}, i_{2},{ }_{-}\right)$is also in the lfp. By the definition of reduce, there is also an atom auxsat $\left(v, i_{1}, i_{2}, P, N,,_{-},-\right)$in the lfp. This atom and the previous reduce predicate imply that $\operatorname{sat}\left(v, i, P, N,_{-},{ }_{-}\right)$exists in the lfp.

Finally we have to show that if $S(v, P, N, C) \neq \emptyset$ then there exists a partition of this set fulfilling the conditions $1-3$ of Property B. Therefore assume that $S=$ $S(v, P, N, C) \neq \emptyset$ and let $S^{\prime}=S\left(v_{1}, P \uplus\{x\}, N, C_{u}\right)$ and $S^{\prime \prime}=S\left(v_{1}, P, N \uplus\right.$ $\left.\{x\}, C_{u}\right)$. Note that by the construction of $S$ it holds that $S=S^{\prime} \cup S^{\prime \prime}$ and $S^{\prime} \cap$ $S^{\prime \prime}=\emptyset$. Therefore at least one of the two sets $S^{\prime}$ and $S^{\prime \prime}$ is not empty. Furthermore by the induction hypothesis, at least one of the following two partitions exists and fulfills the conditions $1-3$ of Property B: $S^{\prime}=\left\{S_{k_{1}}, \ldots, S_{k_{n}}\right\}$ and $S^{\prime \prime}=\left\{S_{l_{1}}, \ldots, S_{l_{m}}\right\}$. Therefore we can define a partition of $S$ as $\left\{S_{k_{1}}, \ldots, S_{k_{n}}, S_{l_{1}}, \ldots, S_{l_{m}}\right\}$.

Now we show that a fact auxsat $\left(v, i_{1}, i_{2}, P, N, C_{u},{ }_{-}\right)$is contained in the lfp iff either $\left(i_{1}, i_{2}\right) \in\left\{\left(k_{1}, 0\right), \ldots,\left(k_{n}, 0\right)\right\}$ or $\left(i_{1}, i_{2}\right) \in\left\{\left(l_{1}, 1\right), \ldots,\left(l_{m}, 1\right)\right\}$. Assume that auxsat $\left(v, i_{1}, i_{2}, P, N, C_{u},-\right)$ is in the lfp. We have to distinguish two cases depending on whether $i_{2}=0$ or $i_{2}=1$. We only treat the first case here, since the second one is symmetrical. In this case a fact $\operatorname{sat}\left(v_{1}, i_{1}, P \uplus\{x\}, N, C_{u},{ }_{-}\right)$has to be in the lfp of the program. Therefore by the induction hypothesis, $i_{1} \in\left\{k_{1}, \ldots, k_{n}\right\}$ and clearly $\left(i_{1}, i_{2}\right) \in\left\{\left(k_{1}, 0\right), \ldots,\left(k_{n}, 0\right)\right\}$. For the other direction, assume that $\left(i_{1}, i_{2}\right) \in$ $\left\{\left(k_{1}, 0\right), \ldots,\left(k_{n}, 0\right)\right\}$ or $\left(i_{1}, i_{2}\right) \in\left\{\left(l_{1}, 1\right), \ldots,\left(l_{m}, 1\right)\right\}$. Again the two cases are similar, hence we only treat the first one here. In this case, by the construction of the partition and the induction hypothesis, a fact $\operatorname{sat}\left(v_{1}, i_{1}, P \uplus\{x\}, N, C_{u},-\right)$ is in the lfp. But then also a fact $\operatorname{auxsat}\left(v, i_{1}, 0, P, N, C_{u},-\right)$ is in the lfp.

Next we show that the fact auxsat $\left(v, i_{1}, i_{2}, P, N, C_{u}, j\right)$ is contained in the lfp iff $\left|S_{i_{1}}\right|=j$. Assume that auxsat $\left(v, i_{1}, i_{2}, P, N, C_{u}, j\right)$ indeed exists in the lfp. Again we only consider the case where $i_{2}=0$. But then the fact $\operatorname{sat}\left(v_{1}, i_{1}, P \uplus\{x\}, N, C_{u}, j\right)$ is also in the lfp and by the induction hypothesis it holds that $\left|S_{i_{1}}\right|=j$. For the other direction assume that $\left|S_{i_{1}}\right|=j$. Depending on the index $i_{1}$ there are again two cases to consider. We treat only the one where $i_{1} \in\left\{k_{1}, \ldots, k_{n}\right\}$. By the induction hypoth- 
esis, the atom $\operatorname{sat}\left(v_{1}, i_{1}, P \uplus\{x\}, N, C_{u}, j\right)$ is in the lfp and therefore also the fact auxsat $\left(v, i_{1}, 0, P, N, C_{u}, j\right)$.

Finally we show that condition 3 of Property B holds when we replace the predicate unsat with auxunsat, i.e. we show that for every partition $\left(P^{\prime}, N^{\prime}\right)$ of $\operatorname{Var}(v)$ and every subset $C_{u}^{\prime} \subseteq C l(v)$, the following three statements are equivalent:

(i) The fact auxunsat $\left(v, i_{1}, i_{2}, P, N, P^{\prime}, N^{\prime}, C_{u}^{\prime}\right)$ is contained in the lfp.

(ii) There exists a $J \in S_{i_{1}}$ and an assignment $J^{\prime} \subset J$, s.t. $J^{\prime} \in S\left(v, P^{\prime}, N^{\prime}, C_{u}^{\prime}\right)$.

(iii) For all $J \in S_{i_{1}}$ there exists an assignment $J^{\prime} \subset J$, s.t. $J^{\prime} \in S\left(v, P^{\prime}, N^{\prime}, C_{u}^{\prime}\right)$.

To facilitate the proof of these equivalences, let $S^{\prime}=S\left(v, P^{\prime}, N^{\prime}, C_{u}^{\prime}\right)$ and $S_{1}^{\prime}=$ $S\left(v_{1}, P^{\prime} \uplus\{x\}, N^{\prime}, C_{u}^{\prime}\right)$. Note that by the construction of these sets, $S_{1}^{\prime} \subseteq S^{\prime}$.

First we show that (i) $\Rightarrow$ (ii). Assume that auxunsat $\left(v, i_{1}, i_{2}, P, N, P^{\prime}, N^{\prime}, C_{u}^{\prime}\right)$ is in the lfp. Again we only consider the case where $i_{2}=0$. Then there is either the fact unsat $\left(v_{1}, i_{1}, P \uplus\{x\}, N, P^{\prime} \uplus\{x\}, N^{\prime}, C_{u}^{\prime}\right)$ or the fact unsat $\left(v_{1}, i_{1}, P \uplus\right.$ $\left.\{x\}, N, P^{\prime}, N^{\prime} \uplus\{x\}, C_{u}^{\prime}\right)$ in the lfp. These two cases are also symmetrical, hence we only consider the first one. By the induction hypothesis, there exists a $J \in S_{i_{1}}$ and an assignment $J^{\prime} \subset J$, s.t. $J^{\prime} \in S_{1}^{\prime}$. Since $S_{1}^{\prime} \subseteq S^{\prime}, J^{\prime} \in S^{\prime}$ and therefore (i) $\Rightarrow$ (ii) holds.

Next we show that (ii) $\Rightarrow$ (iii). Assume there exists a $J \in S_{i_{1}}$ and an assignment $J^{\prime} \subset J$, s.t. $J^{\prime} \in S^{\prime}$. There are four possible cases depending on the truth value of $J(x)$ and $J^{\prime}(x)$. Since they are all symmetrical, we only treat the case where both $J(x)=$ true and $J^{\prime}(x)=$ true. Due to the construction of our partition of $S$, we know that $i_{1} \in\left\{k_{1}, \ldots, k_{n}\right\}$. Furthermore it holds that $J^{\prime} \in S_{1}^{\prime}$. Therefore we can apply the induction hypothesis and hence for all $J \in S_{i_{1}}$ there exists an assignment $J^{\prime} \subset J$, s.t. $J^{\prime} \in S_{1}^{\prime}$. Since $S_{1}^{\prime} \subseteq S^{\prime}, J^{\prime} \in S^{\prime}$ and therefore (ii) $\Rightarrow$ (iii).

Now we show that (iii) $\Rightarrow$ (i). Assume that for all $J \in S_{i_{1}}$ there exists an assignment $J^{\prime} \subset J$, s.t. $J^{\prime} \in S^{\prime}$. Note that for each $J$, the truth value of $J^{\prime}(x)$ is the same, depending only on the partition $\left(P^{\prime}, N^{\prime}\right)$. Therefore there are also four possible cases distinguishable by the truth value of each $J(x)$ and each $J^{\prime}(x)$. We only treat the case where both $J(x)=$ true and $J^{\prime}(x)=$ true. Due to the construction of our partition of $S$, we know that $i_{1} \in\left\{k_{1}, \ldots, k_{n}\right\}$. Since $J^{\prime}(x)=$ true and by the definition of $S$, it holds for each $J^{\prime}$ that $J^{\prime} \in S_{1}^{\prime}$. Hence by the induction hypothesis, the fact unsat $\left(v, i_{1}, P \uplus\{x\}, N, P^{\prime} \uplus\{x\}, N^{\prime}, C_{u}^{\prime}\right)$ is contained in the lfp. Therefore also the predicate auxunsat $\left(v, i_{1}, 0, P, N, P^{\prime}, N^{\prime}, C_{u}^{\prime}\right)$ is in the lfp.

What we have done so far, was basically proving the conditions $1-3$ of Property $\mathrm{B}$ for the predicates auxsat and auxunsat. Note that by the definition of the predicate reduce, the partition $\left\{S_{k_{1}}, \ldots, S_{k_{n}}, S_{l_{1}}, \ldots, S_{l_{m}}\right\}$ of $S$ is transformed into the smallest possible new partition $\left\{S_{1}, \ldots, S_{n^{\prime}}\right\}$ still satisfying the conditions $1-3$ of Property B by combining sets $S_{i_{1}}, S_{i_{2}}, \ldots$ to a new set $S_{i}$ with $i \in\left\{1, \ldots, n^{\prime}\right\}$. Therefore Property $\mathrm{B}$ also holds for the predicates sat and unsat.

The proof of the induction step for the other node types works analogously and is therefore omitted.

\section{Auxiliary Predicates for the \#Horn-Abduction Program}

A datalog implementation of the predicate reduce for the \#HORN-ABDUCTION program plus several auxiliary predicates is given in Figure 7 . The idea of the predicate reduce $\left(v, S, i, i_{1}, i_{2}, j\right)$ is basically the same as for the corresponding predicate of the 
\#CIRCUMSCRIPTION program. We want to detect "equal" pairs of indices $\left(i_{1}, i_{2}\right)$ and map them to a new index $i$. For given values of $v, S$, two pairs $\left(i_{1}, i_{2}\right)$ and $\left(i_{1}^{\prime}, i_{2}^{\prime}\right)$ are equal iff it holds that a fact $\operatorname{aux}\left(v, S, i_{1}, i_{2}, C^{o}, R C, \Delta C, R O, j\right)$ is in the lfp iff $\operatorname{aux}\left(v, S, i_{1}^{\prime}, i_{2}^{\prime}, C^{o}, R C, \Delta C, R O, j^{\prime}\right)$ is in the lfp.

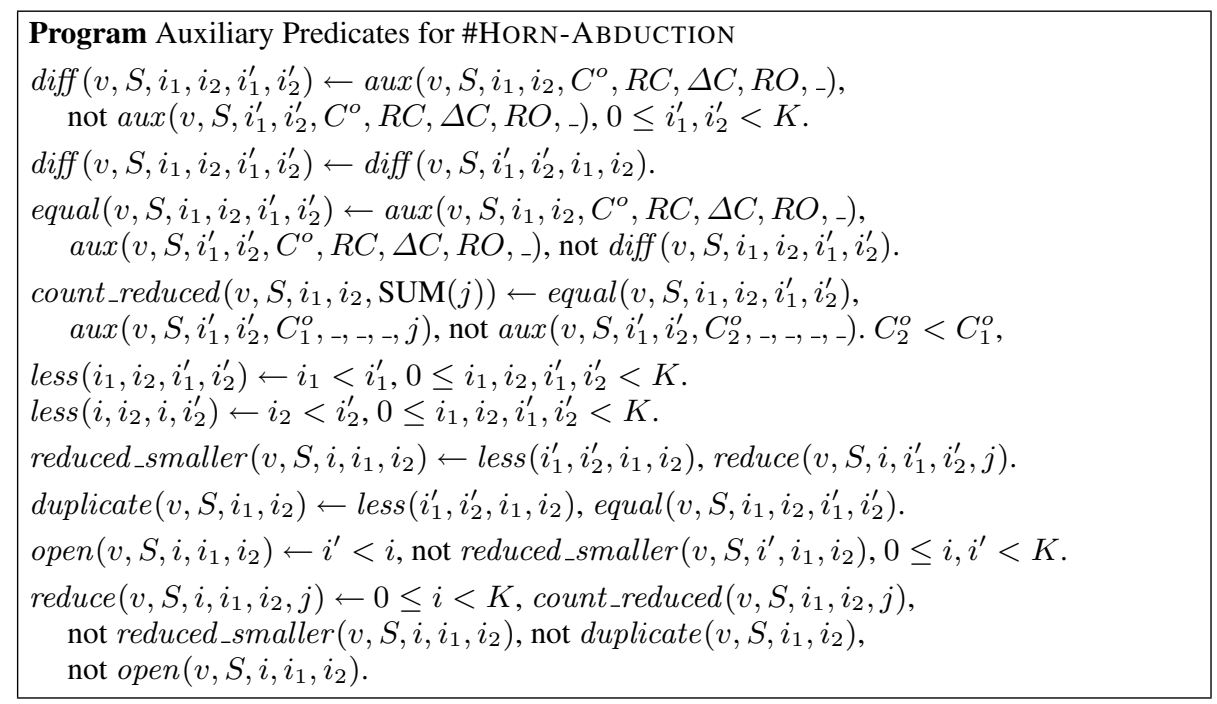

Fig. 7. reduce plus auxiliary predicates for \#HORN-ABDUCTION.

The intended meaning of the auxiliary predicates is the same as for the \#CIRCUMSCRIPTION program except for the fact count_reduced. Since it is possible that for given values of $v, S, i_{1}, i_{2}$ the lfp contains multiple atoms aux $\left(v, S, i_{1}, i_{2}, C^{o}, \ldots\right)$ with different $C^{o}$, we have to ensure that we count only one of them. Therefore the summation is done over those facts with the smallest $C^{o}$ according to some lexicographical ordering. 